\title{
Simultaneous Buffer Insertion and Wire Sizing Considering Systematic CMP Variation and Random $L_{\text {eff }}$ Variation
}

\author{
Lei He, Member, IEEE, Andrew B. Kahng, Member, IEEE, King Ho Tam, and Jinjun Xiong, Member, IEEE
}

\begin{abstract}
This paper presents extensions of the dynamicprogramming (DP) framework to consider buffer insertion and wire-sizing under effects of process variation. We study the effectiveness of this approach to reduce timing impact caused by chemical-mechanical planarization (CMP)-induced systematic variation and random $L_{\text {eff }}$ process variation in devices. We first present a quantitative study on the impact of CMP to interconnect parasitics. We then introduce a simple extension to handle CMP effects in the buffer insertion and wire sizing problem by simultaneously considering fill insertion (SBWF). We also tackle the same problem but with random $L_{\text {eff }}$ process variation (vSBWF) by incorporating statistical timing into the DP framework. We develop an efficient yet accurate heuristic pruning rule to approximate the computationally expensive statistical problem. Experiments under conservative assumption on process variation show that SBWF algorithm obtains $1.6 \%$ timing improvement over the variationunaware solution. Moreover, our statistical vSBWF algorithm results in $43.1 \%$ yield improvement on average. We also show that our approaches have polynomial time complexity with respect to the net-size. The proposed extensions on the DP framework is orthogonal to other power/area-constrained problems under the same framework, which has been extensively studied in the literature.
\end{abstract}

Index Terms-Buffering, dummy fill insertion, fill patterns, interconnect optimization, process variation, random $L_{\text {eff }}$ variation, systematic CMP variation, wire sizing.

\section{INTRODUCTION}

D ESIGN uncertainty in nanometer technology nodes threatens cost-effectiveness of high-performance circuit manufacturing processes. Design uncertainty renders itself in the forms of systematic manufacturing process variation and random process variations due to small geometric dimensions [1]. Considered as one of the most significant source of systematic variation, chemical-mechanical planarization (CMP) is an enabling manufacturing process to achieve uniformity of di-

Manuscript received March 2, 2005; revised June 1, 2005 and March 2, 2006. The work at University of California, Los Angeles (UCLA), was supported in part by the National Science Foundation (NSF) under CAREER award CCR0401682, SRC grant 1100, a UC MICRO grant sponsored by Analog Devices, Fujitsu Laboratories of America, Intel and LSI Logic, and a Faculty Partner Award by IBM. The work at UCSD was supported in part by the MARCO Gigascale Silicon Research Center and the NSF. This paper was recommended by Associate Editor S. Sapatnekar.

L. He, K. H. Tam, and J. Xiong are with the Electrical Engineering Department, University of California, Los Angeles, CA 90095 USA (e-mail: lhe@ee.ucla.edu; ktam@ee.ucla.edu; jinjun@ee.ucla.edu).

A. B. Kahng was with the Electrical and Computer Engineering Department, University of California, San Diego, CA 92093 USA. He is now with Blaze DFM, Inc., Sunnyvale, CA 94089 USA (e-mail: abk@ ucsd.edu).

Digital Object Identifier 10.1109/TCAD.2006.884869 electric and conductor height in back-end-of-line process step. CMP introduces systematic design variations due to dummy fill insertion [2] and dishing and erosion [3]. On the other hand, channel length of a transistor $\left(L_{\text {eff }}\right)$ subjects to random variation as pointed out by [4]. Such variation has great impact on buffered interconnect timing as the buffers' driving strength depends strongly on $L_{\text {eff }}$. As a result of combined systematic and random variations, it is unclear whether interconnects designed from variation-unaware design automation tools live up to the timing yield that we expect by means of static timing analysis. This paper studies the buffer insertion and wire-sizing problem, which is a classical physical design problem, by proposing and experimenting intuitive and efficient ways to deal with process variation.

To deal with systematic variation, it is important to understand the nature and properties of the variation source and its correlation to the design. In the case of CMP, it is understood that dummy fill insertion for CMP planarization changes interconnect capacitance, and that different dummy fill pattern brings different changes. Moreover, metal loss due to uneven polishing, which is dubbed dishing and erosion in CMP terminology, adds to variation in interconnect resistance. However, there is no extensive and quantitative study in the literature on the interconnect performance variation due to CMP. In [5], it assumes only one regular fill pattern array and shows that the increase of interconnect capacitance due to such a fill pattern cannot be ignored for interconnect optimization. In [6], the authors considered the variation of total capacitance due to the Boolean-based placement of dummy fills and have shown that up to $25 \%$ variation is possible, albeit with only one fill pattern. In [7], it was proposed to examine the impact due to different fill patterns, however, no quantitative experiment results have been reported.

Research has started emerging on circuit optimization for yield improvement considering process variations. Statistical timing analysis [8]-[10] has been studied recently, but results mainly focus on analysis rather than design. Most statistical circuit optimization works focus on solving the gate-sizing problem. In [11], it introduces modification to the nonlinear programming formulation for the gate-sizing problem through iterative delay constraint adjustment. In [12], it is similar except that the modification is based on scaling the objective function with a "dis-utility" function, which is an ad hoc metric that reflects the "spread" of the overall timing distribution. More recently, [13] proposes a statistical sensitivity-based gate-sizing algorithm which is based on bound computation of probability. 
All these works either assume delay distributions as Gaussian or do not compute accurate cumulative distribution function (CDF). Another recent work [14] presents a buffer insertion methodology in a routing tree, which considers the uncertainty in wire-length estimation but not process variations such as CMP effects and $L_{\text {eff }}$ variation.

This paper first quantitatively studies interconnect parasitic variations due to CMP effects. Specifically, we study different fill patterns that are "equivalent" with respect to foundry rules, and dishing and erosion of conductors and dielectric similar to those predicted by International Technology Roadmap for Semiconductors (ITRS) [15] (Section II). We then present our extension of the dynamic-programming (DP) framework [16] which solves the simultaneous buffer insertion and wire sizing problem [17] under CMP-induced systematic variation and random $L_{\text {eff }}$ variation. To perform optimization under CMP effects, fill pattern design must be considered simultaneously with buffers and wire sizes, and we name the resulting problem as SBWF (Section III). We then discuss the SBWF problem which also considers random $L_{\text {eff }}$ variation (vSBWF) by designing with statistical timing (Section IV). We propose a few techniques which accurately and efficiently handle statistical timing that avoids the exponential runtime complexity. We conclude this paper with discussion of our future research (Section V).

\section{MODELING OF CMP VARIATION}

This section describes the effect of dummy fill insertion, dishing, and erosion on interconnect parasitics as a result of CMP. To minimize dishing and erosion, foundries require dummy fill insertion to even out the metal density across the die. However, dummy fill insertion may lead to an increase in parasitic capacitance on interconnects. This section describes the parasitic model and presents statistics of potential impact of CMP on parasitic capacitance and resistance.

\section{A. Fill Patterns Exploration}

We explore a wide range of design-rule-check (DRC) correct fill patterns. We assume rectangular, isothetic fill features aligned horizontally and vertically between two adjacent interconnects as shown in Fig. 1, which are sandwiched between upper and lower ground planes. In the figure, conductors $A$ and $B$ are active interconnects and the metal shapes between them is dummy fill. We assume all dummy fill is implemented as floating metals in the final layout, as floating dummy fill are preferred for most application-specified integrated circuit designs due to the short design time and considerable area to be filled [5], [18]. Each distinct, DRC-correct fill pattern $P\left(M, N, W_{i}, L_{j}, S_{x, i}, S_{y, j}\right)$ is specified by:

1) the number of fill rows $(M)$ and columns $(N)$;

2) the series of widths $\left\{W_{i}\right\}_{i=1, \ldots, N}$ and lengths $\left\{L_{j}\right\}_{j=1, \ldots, M}$ of fills, where $W_{i} \in\left[\overline{W_{l}}, \overline{W_{u}}\right] \forall i$;

3) the series of horizontal and vertical spacings between fills, $\left\{S_{x, i}\right\}_{i=1, \ldots, N}$ and $\left\{S_{y, j}\right\}_{j=1, \ldots, M}$, where $\left\{S_{x, i}\right\}_{i=2, \ldots, N-1}$ are at least $\overline{S_{l}}$ and those between metal and dummy fill $\left\{S_{x, i}\right\}_{i=1, N-1}$ are at least $\overline{S_{d}}$.

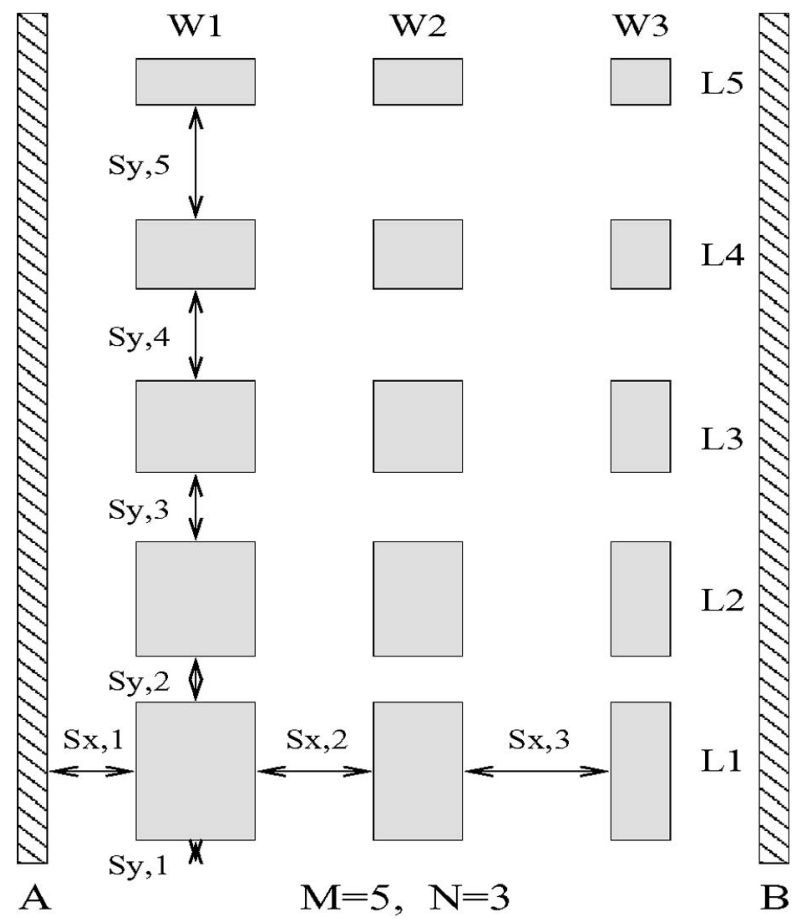

Fig. 1. Fill pattern definition.

Foundries require the effective metal density $\rho_{\mathrm{Cu}}$ throughout the die. We express the actual amount of metal fill needed between interconnect in terms of local metal density $\rho_{\mathrm{f}}$.

Definition 1: Effective metal density $\rho_{\mathrm{Cu}}$-the proportion of the area in a planarization window [3] that all metal features (interconnect + dummy fill metal) occupies-which is usually a hard requirement from the foundry [2], [19].

Definition 2: Local metal density $\rho_{\mathrm{f}}$-the proportion of the oxide area between two neighboring interconnects that dummy fill metal occupies-which is found by either rulebased method in the industry or by the recently proposed model-based method [20] to achieve $\rho_{\mathrm{Cu}} \cdot{ }^{1}$

With the above definitions, we can therefore derive the width of length of the dummy metal fill features by $\rho_{\mathrm{f}} \cdot S_{A, B}=$ $\sum_{i} W_{i} \cdot \sum_{j} L_{j}=W_{b} \cdot L_{b}$, where $S_{A, B}$ is the space between interconnect $A$ and $B$, and $W_{b}$ and $L_{b}$ are the total fill width budget and length budget, respectively. Finding a valid fill pattern is equivalent to distributing the budgets of $W_{b}, L_{b}, S_{x, b}$, and $S_{y, b}$ among their respective series $\left\{W_{i}\right\},\left\{L_{j}\right\},\left\{S_{x, i}\right\}$, and $\left\{S_{y, j}\right\}$, which also determines $M$ and $N$.

To understand the impact of different dummy fill pattern on variation of parasitic capacitance, we explore many different fill patterns, each of which satisfies the aforementioned DRC restrictions and its metal fill target. Fig. 2 shows the $x$-crosssectional views of three different fill patterns. We plot $f(z)$ as the width of each dummy fill feature against the position of the space that we want to fill. By constraining the area under $f(z)$ to the budget width $W_{b}$, we try numerous shapes of $f(z)$, among which we discard those that violate the DRC restrictions. We apply similar enumeration to the other side

\footnotetext{
${ }^{1}$ Although the cited reference refers to a shallow-trench isolation process, the underlying polishing process resembles that in copper CMP.
} 


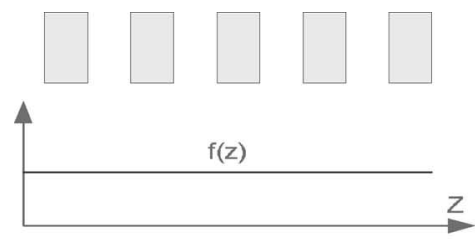

(1)

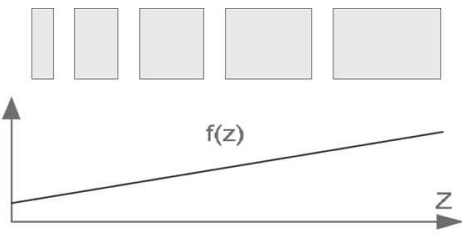

(2)

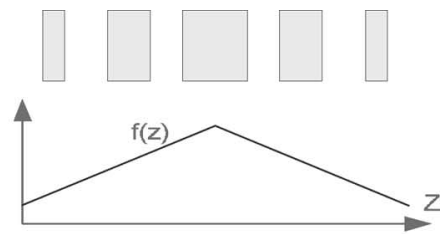

(3)

Fig. 2. Examples of cross-sectional profile function $f(z)$.

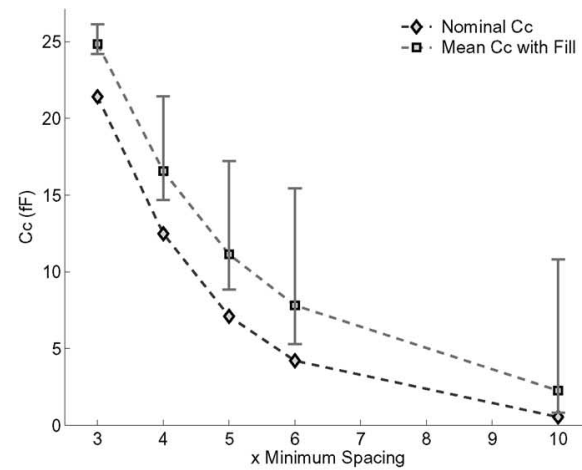

(a)

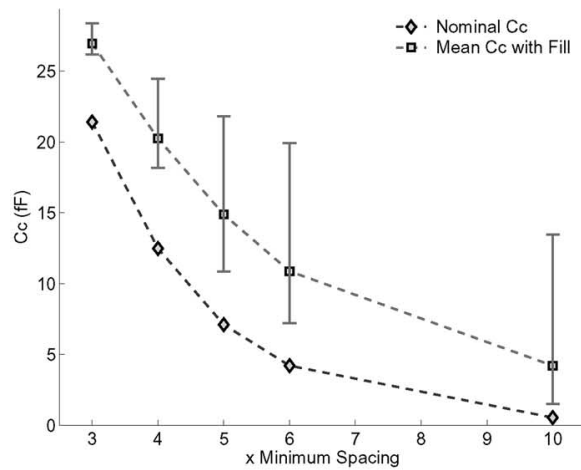

(b)

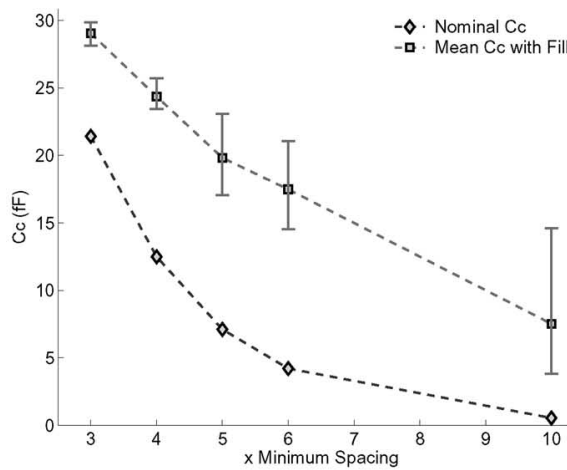

(c)

Fig. 3. Distribution of coupling capacitance $C_{\mathrm{c}}$. (a) $\rho_{\mathrm{f}}=0.3$. (b) $\rho_{\mathrm{f}}=0.5$. (c) $\rho_{\mathrm{f}}=0.7$.

of the cross section (i.e., $y$-direction) to obtain a complete exploration of fill patterns.

\section{B. Fill Pattern Induced Variation}

We consider the coupling capacitance $\left(C_{\mathrm{c}}\right)$ between active interconnects and total capacitance $\left(C_{\mathrm{s}}\right)$ of an interconnect, which is the sum of $C_{\mathrm{c}}$, area capacitance, and fringe capacitance. Inserting dummy fill between signal wires effectively brings the signal wires closer together, where they couple stronger with each other through the floating metal. However, the floating metal has coupling to above and below layers which may act as alternative paths for coupling currents between these signal lines. We use QuickCap [21] to extract the effective $C_{\mathrm{c}}$, which gives the capacitance that achieves the same coupling effect by replacing the dummy fill structure with a simple capacitor between the signal lines. The on-chip interconnect is modeled as a stripline where the interconnect layer is sandwiched between two ground planes. We study global interconnects in the ITRS 65-nm technology node [15] with various fill pattern that we generate from Section II-A. For each layout, the interconnect width is set to the minimum width while the spacing between two active interconnects varies from $3 \times$ to $10 \times$ minimum spacing. ${ }^{2}$ Interconnect length is $1000 \mu \mathrm{m}$ for all layouts. We extract and compare the nominal (i.e., without dummy fill) and the CMP-impacted (i.e., with dummy fill) $C_{\mathrm{c}}$ and $C_{\mathrm{s}}$.

Fig. 3 plots the variation of coupling capacitance $C_{\mathrm{c}}$ due to dummy fill insertion. We examine the cases where $\rho_{\mathrm{f}}=0.3$, 0.5 , and 0.7 . We vary the spacing between interconnects from $3 \times$ to $10 \times$ minimum spacing. The curves with diamond sym-

\footnotetext{
${ }^{2}$ To allow fill insertion between active interconnect without DRC violation, wire spacing has to be at least $3 \times$ the minimum.
}

bols are the nominal $C_{\mathrm{c}}$ without fill insertion. The curves with square symbols represent the mean values of the effective $C_{\mathrm{c}}$ under dummy fill insertion. The ranges of $C_{\mathrm{c}}$ due to different dummy fill patterns are represented by their respective maximum and minimum values among all the fill patterns, which are shown by the vertical bar on each square symbol. We observe that: 1) different fill patterns result in coupling capacitance variation, which can be up to $10 \%$ at $3 \times$ spacing and more than double at $6 \times$ spacing; 2) fill insertion always increases the coupling capacitance when compared to the nominal case (i.e., without dummy fill); and 3) the gap between the nominal $C_{\mathrm{c}}$ curve and the mean value $C_{\mathrm{c}}$ curve increases with metal fill density $\rho_{\mathrm{f}}$.

To study the relative importance of the coupling capacitance variation versus the total capacitance variation due to fill insertion, in Fig. 4, we plot the percentage of $C_{\mathrm{c}}$ over $C_{\mathrm{s}}$ with respect to different local metal densities $\rho_{\mathrm{f}}(0.1$ to 0.7$)$ between active interconnects with $3 \times, 5 \times$, and $10 \times$ minimum spacing. The gap between the maximum and minimum percentage curves shows the potential variation due to fill insertion. We see that: 1) fill insertion increases the percentage of $C_{\mathrm{c}} / C_{\mathrm{s}}$ ratio in all different metal densities and interconnect spacing and 2) variation of $C_{\mathrm{c}} / C_{\mathrm{s}}$ increases with metal spacing and slightly with metal density. The exact impact of these variation on the actual delay remains to be seen; however, since large metal spacing undermines the significance of $C_{\mathrm{c}}$, it affects its variation.

\section{Dishing and Erosion Induced Variation}

Fig. 5 illustrates dishing and erosion phenomena due to CMP [22]. Step height is defined as the difference of height between different area on the surface of the wafer. Dishing is a special case of step height that it specifically refers to the difference 


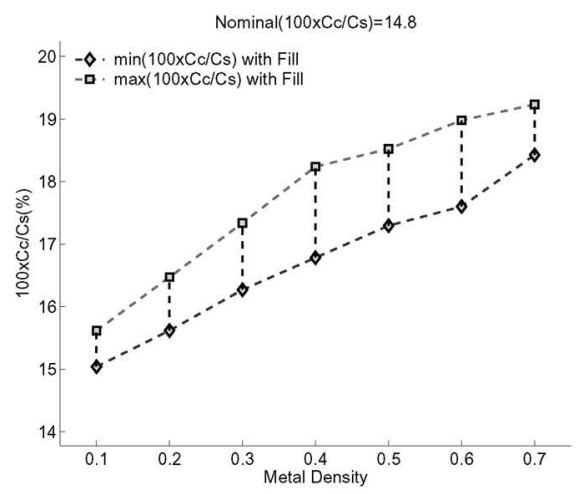

(a)

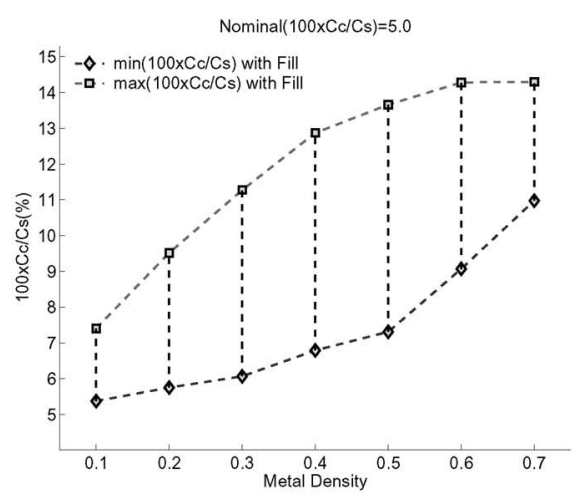

(b)

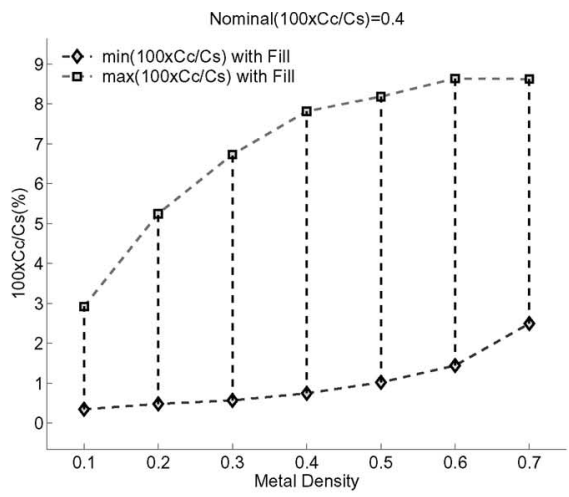

(c)

Fig. 4. Percentage of $C_{\mathrm{c}}$ over $C_{\mathrm{s}}$ for different local metal density requirement $\rho_{\mathrm{f}}$. (a) $3 \times$ minimum spacing. (b) $5 \times$ minimum spacing. (c) $10 \times$ minimum spacing.

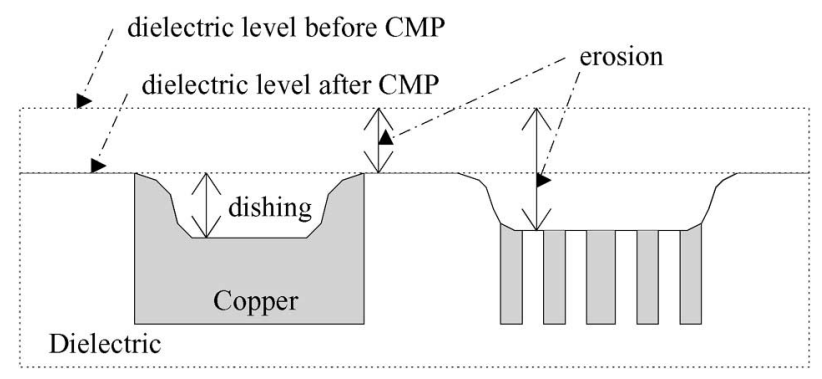

Fig. 5. Dishing and erosion in copper CMP.

between the height of the copper in the trench, which defines the metal interconnect, and that of the dielectric in the space surrounding the trenches. Erosion is defined as the difference between the dielectric thickness before CMP and that after CMP. The sum of dishing and erosion is the total loss of metal thickness.

We employ the dishing and erosion model in [22], which is a closed-form solution of a differential equation set, to calculate postmultistep CMP interconnect geometries. ${ }^{3}$ During interconnect formation, trenches are etched on the oxide, followed by barrier deposition on the etched surface. Then, a thick layer of copper is deposited. CMP removes both the bulk copper above the trenches and the barrier on the area between the trenches. The multistep model consists of three phases. We assume that step 1) eliminates all the local step heights before touching the raised area and is therefore irrelevant to the modeling of dishing and erosion. We also assume that step 2) completely removes all the remaining copper so that there is no dishing and erosion at the moment when the polishing pad reaches the barrier on the raised area. We use the same assumption as in Gbondo-Tugbawa's model [22] that the polishing time of step 2) after reaching the barrier layer is $20 \mathrm{~s}$ and that of the entire step 3) is $65 \mathrm{~s}$.

To show the potential impact of dishing and erosion on signal wire's parasitics, we apply the model and measure the resistance and capacitance of the middle interconnect in a strip-line

\footnotetext{
${ }^{3}$ To the best of our knowledge, this is the only published copper CMP model with process parameters in the literature, which serves as a reasonably assumed input to this paper. Our subsequent process variation aware methodologies do not necessarily depend on these assumed CMP parameters.
}

structure. ${ }^{4}$ Table I shows the RC parasitics for a $1000-\mu \mathrm{m}$-long global interconnect bus structure under the $65-\mathrm{nm}$ technology node. $R_{0}$ is the resistance computed from the geometry values obtained from ITRS specifications, i.e., dishing and erosion effects are not taken into account. $R_{\mathrm{f}}$ is the resistance after fill insertion which fulfills $50 \%$ metal density requirement (i.e., $\left.\rho_{\mathrm{Cu}}=0.5\right)$. Based on this, we include the metal loss due to dishing and erosion when computing $R_{\mathrm{f}}$. From Table I, we can see that resistance variation due to dishing and erosion is significant, and that resistance is always increasing, potentially by more than $30 \%$. As width increases, the resistance variation becomes increasingly severe. For example, when conductor width increases from 0.24 to $2.61 \mu \mathrm{m}$, the resistance variation increases from $29 \%$ to $31 \%$.

All capacitance values in Table I are extracted using QuickCap [21]. $C_{\mathrm{c}, 0}$ and $C_{\mathrm{s}, 0}$ are the coupling and total capacitance without considering fill insertion or dishing and erosion effects. $C_{\mathrm{c}, 1}$ and $C_{\mathrm{s}, 1}$ are the coupling and total capacitance for the same assumed structure as in Section II-B, taking geometry variations due to dishing and erosion (but no fill insertion) into account. Finally, $C_{\mathrm{c}, \mathrm{f}}$ and $C_{\mathrm{s}, \mathrm{f}}$ are the effective coupling and total capacitance when effects due to dummy fill, dishing and erosion are all taken into consideration. The percentages in the brackets show the relative changes from values which do not consider any CMP effect (columns 3, 5, and 6). From Table I, we observe that dishing and erosion alone merely have any impact on capacitance. In light of these results, we do not consider dishing and erosion effects on capacitance in our subsequent discussion.

\section{Table-Based Fill Pattern Look-Up and RC Model}

Based upon this paper of CMP-induced $R C$ parasitic variations, we tabulate the extracted capacitance in a table indexed by active interconnect width, spacing, and local metal density under an optimized fill pattern. Note that varying metal spacing affects the local metal density requirement. During interconnect optimization, each enumerated spacing option requires

\footnotetext{
${ }^{4} \mathrm{We}$ are interested in global wires that are $\leq 2-\mu \mathrm{m}$ wide as predicted in 65 -nm ITRS process [15]. Wider lines like power grids, which are out of the scope of this paper, require slotting to prevent "lift-off" in CMP [23].
} 
TABLE I

RC PARASITIC COMPARISON FOR 65-nm GLOBAL INTERCONNECTS

\begin{tabular}{|c|c|c|c|c|c|c|c|c|c|}
\hline Width & Space & wo/CMP & w/CMP & \multicolumn{2}{|c|}{ wo/CMP } & \multicolumn{2}{c|}{ Dishing/Erosion } & \multicolumn{2}{c|}{ Fill+Dishing/Erosion } \\
\cline { 3 - 9 }$\mu m$ & $\mu m$ & $R_{0}(\Omega)$ & $R_{f}(\Omega)$ & $C_{c, 0}$ & $C_{s, 0}$ & $C_{c, 1}(\Delta \%)$ & $C_{s, 1}(\Delta \%)$ & $C_{c, f}(\Delta \%)$ & $C_{s, f}(\Delta \%)$ \\
\hline 0.24 & 0.95 & 186 & $239(28.7 \%)$ & 25.16 & 286.06 & $24.48(-2.63 \%)$ & $285.12(-0.33 \%)$ & $33.48(33.06 \%)$ & $285.77(-0.11 \%)$ \\
2.61 & 0.95 & 16.9 & $22.1(30.6 \%)$ & 26.06 & 966.82 & $25.06(-3.78 \%)$ & $964.98(-0.19 \%)$ & $32.90(26.33 \%)$ & $953.71(-1.35 \%)$ \\
0.24 & 1.43 & 186 & $239(28.8 \%)$ & 8.35 & 283.75 & $8.57(2.54 \%)$ & $283.39(-0.13 \%)$ & $20.27(142.71 \%)$ & $289.12(1.88 \%)$ \\
2.61 & 1.43 & 16.9 & $22.1(30.9 \%)$ & 8.68 & 956.84 & $8.32(-4.35 \%)$ & $954.04(-0.29 \%)$ & $21.02(141.81 \%)$ & $960.34(0.36 \%)$ \\
\hline
\end{tabular}

an appropriate adjustment to the required local metal density. Therefore, fill pattern and $R C$ of all combinations of spacing and local metal density have to be recorded in the table to accommodate any arbitrary spacing and adjusted local metal density. Moreover, as different fill patterns under the same local metal density result in different capacitance values as shown in Section II-B, each table entry only saves the fill pattern and the resulting capacitance under the best fill pattern, which gives the minimum $C_{\mathrm{c}}$ among all patterns. We use the briefly discussed model in Section II-C to compute the resistance under dishing and erosion effects. In the following, we call the resulting $R C$ models as CMP-aware $R C$ parasitic models. In contrast, interconnect parasitics without consideration of fill pattern insertion, dishing, or erosion effects are called CMPoblivious $R C$ model.

\section{CMP-AWARE BUfFER INSERTION AND WIRE SizING}

In this section, we study the problem of simultaneous buffer insertion and wire sizing (SBW) to examine the impact of CMP on interconnect design. ${ }^{5}$ We propose an extension to the popular DP-based SBW algorithm [16], [17] to solve the SBW and the fill insertion problem simultaneously, and we denote it as SBWF. In contrast, current designers first solve the SBW problem with CMP-oblivious $R C$ and then hand the design off for a postlayout processing step. This second step inserts dummy fill metal into the wire space to satisfy the local metal density requirement defined in Section II-A. We use this twostep approach as our baseline for comparison, which is denoted as SBW + Fill.

\section{A. Problem Formulation}

Consider a routing tree $T(V, E)$, where $V$ consists of a source node $n_{\text {src }}$, sink nodes $\left\{n_{\mathrm{s}}\right\}$, and Steiner points $\left\{n_{\mathrm{p}}\right\}$, and $E$ is the set of directed edges (wires) that connect the nodes in $V$. The SBWF problem is to find an assignment of buffer insertion, buffer sizing, wire sizing, and dummy fill insertion, such that the required arrival time (RAT) is maximized at $n_{\mathrm{src}}$, subject to: 1) the slew rate constraint $\eta$ at all $n_{\mathrm{s}}$ and buffers' inputs and 2) the effective metal density requirement $\rho_{\mathrm{Cu}}$ for CMP planarization.

We characterize the source $n_{\text {src }}$ by a driving resistance $R_{\mathrm{src}}$, each sink $n_{\mathrm{s}}$ by a loading capacitance $C_{n}^{\mathrm{s}}$ and a $\mathrm{RAT}_{\mathrm{s}}$. We associate each edge $e_{i, j}$ with two center-to-edge wire widths $w_{1}$ and $w_{2}$ as illustrated in Fig. 6. To respect the design rules, we restrict $w_{k} \in\left\{0.5 \cdot \check{w}, 1.5 \cdot \check{w}, \ldots, s_{k}-\check{w}\right\}$, where $k=1,2, \check{w}$ is the minimum wire width allowed at the global metal level

\footnotetext{
${ }^{5}$ The asymmetric wire sizing problem was first proposed in [17], which does not consider the CMP-induced variation.
}

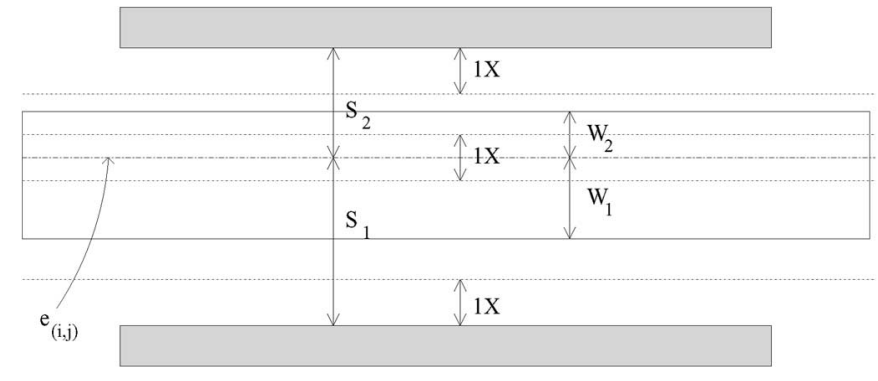

Fig. 6. Illustration of asymmetric wire sizing.

and $s_{k}$ is the spacing from the center line to the edges of its two nearest neighboring wires. For every edge $e_{i, j}$, we define the potential buffer insertion site at the point closest to the node $v_{i}$. The buffer receives input from node $v_{i}$ and drives edge $e_{i, j}$ and the downstream subtree rooted at node $v_{j}$. We express the size of buffer $S_{\text {buf }}$ in discrete multiples of the minimum-sized buffers. All buffers are two-stage cascaded inverters.

\section{B. Slew Rate Constrained SBW Algorithm}

The slew rate constrained SBW algorithm largely follows the DP framework of [24], where buffer insertion and asymmetric wire sizing are determined in a bottom-up (sink-to-source) recursive fashion. To obtain the optimal solution at the source in a deterministic buffer insertion regime, partial solutions $\operatorname{sol}_{n}$ at node $n$ (i.e., partial buffer placement and wire width assignment for the subtree rooted at node $n$ ) must keep track of the downstream capacitance $C_{n}$ and the arrival time $\mathrm{RAT}_{n}$ associated with $\operatorname{sol}_{n}$. The arrival time $\mathrm{RAT}_{n}$ at node $n$ is defined by

$$
\operatorname{RAT}_{n}=\min _{n_{i} \in\left\{n_{\mathrm{s}}\right\}}\left(\mathrm{RAT}_{i}-d\left(n_{i}, n\right)\right)
$$

where $d\left(n_{i}, n\right)$ is the delay from the node $n_{i}$ to node $n, \mathrm{RAT}_{i}$ is the RAT at node $n_{i}$ and $\left\{n_{\mathrm{s}}\right\}$ is the set of all sink nodes. We use the first-order Elmore delay model and slew rate model [25] in our current implementation due to their high fidelity over real design metrics. We update the RAT ${ }_{n}$ of each solution $\operatorname{sol}_{n}$ at node $n$ by

$$
\begin{aligned}
\operatorname{RAT}_{n}=\mathrm{RAT}_{n}^{\text {old }}-r_{n, v} \cdot C_{n}- & 0.5 \cdot r_{n, v} \cdot c_{n, v} \\
& -d_{\text {buf }}-R_{\text {eff }} \cdot\left(L_{n}+c_{n, v}\right)
\end{aligned}
$$

where $r_{n, v}$ and $c_{n, v}$ are the resistance and capacitance of edge $e_{n, v}$, respectively. $d_{\text {buf }}$ and $R_{\text {eff }}$ are buffer intrinsic delay and output resistance, which are both functions of buffer size $S_{\text {buf }}$. We use Bakoglu's slew rate metric [25] given by $\ln 9 \cdot d_{T}^{n}$, where $d_{T}^{n}$ is the maximum delay from the output of buffer at node $n$ to the inputs of other immediate buffers or sinks in the subtree $T_{n}$ rooted at $n$. Note that the above can be replaced 
by other more accurate delay [26] and slew [27] metrics which consider higher order moments.

The overall time complexity of the SBW + Fill algorithm is $O\left(|V|^{2} \cdot c_{\max } \cdot\left(\left|S_{\text {buff }}\right|+\left|S_{\text {wire }}\right|\right)\right)$, where $\left|S_{\text {wire }}\right|$ is the number of available choices of wire widths, $|V|$ is the number of nodes in the interconnect tree, $c_{\max }$ is the maximum possible capacitance value carried by any partial solutions and $\left|S_{\mathrm{buf}}\right|$ is the number of possible sizes for buffers [24]. The complexity depends on $c_{\max }$ if we prune inferior solutions in $\mathrm{SOL}_{n}$ for each node $n$. A solution $\mathrm{sol}_{1}$ is said to be inferior to (or dominated by) another solution $\mathrm{sol}_{2}$ if $C_{\text {sol }}^{1} \geq C_{\text {sol }}^{2}$ and $\mathrm{RAT}_{\text {sol }}^{1} \leq \mathrm{RAT}_{\text {so }}^{2}$. With wire sizing, $c_{\max }$ can go exponential but is in fact upper bounded. The slew rate bound virtually limits the distance that a wire can run without buffering, which therefore limits the maximum downstream capacitance $c_{\max }$ seen from any node.

\section{Extension to $S B W$ and $S B W F$}

We extend the SBW + Fill algorithm to solve the SBWF problem, and such an approach is denoted as SBWF wherever there is no ambiguity. SBWF uses the CMP-aware table-based $R C$ model from Section II-D for delay and slew rate calculation while solving the slew rate constrained SBW problem. For every edge $e_{i, j}$, we specify two local dummy fill density requirements $\rho_{\mathrm{f}}^{1}$ and $\rho_{\mathrm{f}}^{2}$ at minimum wire width in order to satisfy the effective metal density target $\rho_{\mathrm{Cu}}$, as defined in Section II-A. The required $\rho_{\mathrm{f}}^{1}$ and $\rho_{\mathrm{f}}^{2}$ can be determined from algorithms such as [20]. Note that increasing wire width decreases the amount of dummy fill metal needed between wire space, which necessitates the adjustment to the required local metal densities. At each enumeration of wire spacing option, the SBWF algorithm makes an adjustment to $\rho_{\mathrm{f}}^{1}$ and $\rho_{\mathrm{f}}^{2}$, which are used with the corresponding wire widths and spacing to look up the CMP-aware fill pattern and $R C$ table for the optimized fill pattern and the capacitance values. The algorithm collects all wire sizing and spacing options, each with timing evaluated under an optimized fill pattern. These options are then pruned against each other as in the SBW + Fill algorithm to remove inferior solutions.

Note that the proposed extension is orthogonal to the baseline DP-based framework. This extension brings in the necessary bookkeeping to maintain wire width, spacing, and local metal density requirement, which supports calculation of dishing/ erosion and the table-lookup methodology in Section II-D for optimum dummy fill patterning. This extension can be applied to many other variants of such dynamic-programming framework, which include cost/power consideration and speed-up techniques [24], [28], [29]. ${ }^{6}$

\section{Experiment}

Table II shows the experimental settings used in this paper. We choose typical buffer sizes and wire sizes that are normally used in real designs. Since there is no physical layout information in the original test cases obtained from [31], we

\footnotetext{
${ }^{6}$ There exists a wealth of discussions in the literature about design with cost constraints and algorithmic speedup, which we consider as orthogonal issues and are therefore not discussed in length for conciseness and focus.
}

TABLE II

EXPERIMENTAL SETTINGS

\begin{tabular}{|c|c|}
\hline technology & ITRS 65nm [15] \\
\hline interconnect & global interconnect layer \\
\hline delay model & Elmore delay, $\pi$-model for interconnect \\
\hline slew model & Bakoglu's first order metric [25] \\
\hline power model & dynamic and short-circuit, from SPICE \\
\hline device & BSIM 4 [30] \\
\hline$R_{\text {src }}$ & $100 \Omega$ \\
\hline$L_{\text {sink }} \& R A T_{\text {sink }}$ & $100 p s$ (under CMP-perturbed RC) \\
\hline slew bound $\bar{\eta}$ & $0 \sim 0.8$ (local fill), 0.5 (effective) \\
\hline metal density & $20,40,80,120$ (x min size) \\
\hline$S_{\text {buf }}$ & $1.5 \sim 5.5$ (x min width) \\
\hline$s_{1}, s_{2}$ & $0.5,2.5,4.5$ (x min width) \\
\hline$w_{1}, w_{2}$ & $500 \mu m$ \\
\hline segment length & $\mathrm{r} 1 \sim \mathrm{r} 5:$ clock trees from [31] \\
\hline test cases & $\mathrm{s} 1 \sim \mathrm{s} 10:$ random Steiner trees \\
\hline
\end{tabular}

randomly generate the neighboring wire spacing data and the local metal density requirements for each interconnect in all test cases. We perform experiments on an Intel Xeon $1.9-\mathrm{GHz}$ Linux workstation with 2-Gb of memory.

In order to make a conservative review on the effect of the SBWF methodology, we assume the best possible scenario that designers can account for the effect of CMP in the baseline $\mathrm{SBW}+$ Fill approach. We first assume that designer makes the best effort to introduce the minimum overdesign to the slew rate constraint $\eta$ in order to meet the actual slew rate constraint under CMP-aware parasitics and inserted dummy fill. The first step of the SBW + Fill algorithm always underestimates the slew rate as it does not consider CMP-induced variation on $R C$. The overconstrain rate $\kappa$ is defined as the ratio of the overconstrained slew rate to the actual slew rate constraint. To minimize overdesign, we find $\kappa$ via an expensive binary search, in which each iteration involves an execution of SBW + Fill. In contrast, the proposed SBWF algorithm uses the CMP-aware $R C$ parasitics while solving SBW problem. Therefore, it finds an optimum solution that satisfies the slew rate constraints without repetition. In our current setting, we use $\kappa=0.84$ for $\mathrm{SBW}+$ Fill, which gives maximum slew rates that satisfy the slew rate bound $\eta$ in all test cases. Our second conservative assumption is that the postlayout processing step in SBW + Fill does make an effort to choose the fill pattern that minimizes the increase in capacitance. In contrast, most works in the literature only consider one single pattern [5]-[7], which does not necessarily minimize the impact of fill insertion on parasitics.

Table III compares the experimental results from SBW + Fill and SBWF. The objective in both SBW + Fill and $S B W F$ is to optimize the RAT at the source. We verify both the $\mathrm{SBW}+$ Fill design and the SBWF design under the CMPaware parasitic model. A solution with larger RAT implies smaller delay and is therefore more preferable. Comparing SBW + Fill against SBWF (relative change of values shown in the brackets), we see that SBWF consistently achieves larger RAT for all test cases and the average increase is $1.6 \%$. Accounting for CMP variation by SBWF comes at a cost of having an average of $5.0 \%$ increase in wiring area, although buffer area drops by $4.9 \%$ on average. Overconstraining the slew rate in SBW + Fill causes excessive buffer insertion in SBW + Fill 
TABLE III

EXPERIMENTAL RESUlt From SBW + Fill AND SBWF Verified UNDER CMP-PERTURbed $R C$

\begin{tabular}{|c|c|c|c|c|c|c|c|c|c|c|c|c|}
\hline & & & \multicolumn{5}{|c|}{ SBW + Fill $(\kappa=0.84)$} & \multicolumn{5}{|c|}{$S B W F$} \\
\hline $\begin{array}{l}\text { test- } \\
\text { case }\end{array}$ & $\begin{array}{c}\text { wire } \\
\text { length } \\
(m)\end{array}$ & $\begin{array}{c}\# \\
\text { sink }\end{array}$ & $\begin{array}{c}\text { wire } \\
\text { area } \\
\left(m m^{2}\right)\end{array}$ & $\begin{array}{l}\text { buffer } \\
\text { area } \\
\text { (x min) }\end{array}$ & $\begin{array}{l}\text { RAT } \\
(p s)\end{array}$ & $\begin{array}{c}\text { power } \\
(p J)\end{array}$ & $\begin{array}{l}\text { run- } \\
\text { time } \\
(s)\end{array}$ & $\begin{array}{c}\text { wire area } \\
\left(\mathrm{mm}^{2}\right) \\
(\Delta \%)\end{array}$ & $\begin{array}{c}\text { buffer area } \\
(\mathrm{x} \text { min }) \\
(\Delta \%)\end{array}$ & $\begin{array}{c}\text { RAT } \\
(p s) \\
(\Delta \%)\end{array}$ & $\begin{array}{l}\text { power } \\
(p J) \\
(\Delta \%)\end{array}$ & $\begin{array}{l}\text { run- } \\
\text { time } \\
(s)\end{array}$ \\
\hline s1 & 0.03 & 19 & 0.10 & 2920 & -1007 & 22 & 0 & $0.10(0.9 \%)$ & $2680(-8.2 \%)$ & $-1001(0.6 \%)$ & $21(-6.0 \%)$ & 0 \\
\hline s2 & 0.04 & 29 & 0.11 & 3420 & -1175 & 26 & 0 & $0.12(2.0 \%)$ & $3140(-8.2 \%)$ & $-1133(3.6 \%)$ & $25(-5.7 \%)$ & 1 \\
\hline s3 & 0.05 & 49 & 0.14 & 4380 & -1589 & 33 & 1 & $0.15(9.5 \%)$ & $4360(-0.5 \%)$ & $-1567(1.3 \%)$ & $34(0.9 \%)$ & 1 \\
\hline s4 & 0.07 & 99 & 0.18 & 6180 & -1386 & 47 & 2 & $0.19(8.0 \%)$ & $6060(-1.9 \%)$ & $-1380(0.4 \%)$ & $46(-0.5 \%)$ & 2 \\
\hline s5 & 0.10 & 199 & 0.26 & 8820 & -2436 & 67 & 4 & $0.27(5.3 \%)$ & $8500(-3.6 \%)$ & $-2409(1.1 \%)$ & $66(-2.1 \%)$ & 5 \\
\hline s6 & 0.13 & 299 & 0.31 & 11720 & -2294 & 88 & 7 & $0.33(5.9 \%)$ & $11020(-6.0 \%)$ & $-2235(2.6 \%)$ & $84(-3.9 \%)$ & 8 \\
\hline s7 & 0.16 & 499 & 0.38 & 15220 & -3794 & 113 & 16 & $0.40(5.1 \%)$ & $14520(-4.6 \%)$ & $-3787(0.2 \%)$ & $110(-3.0 \%)$ & 22 \\
\hline s8 & 0.19 & 699 & 0.43 & 18320 & -3170 & 136 & 37 & $0.45(4.7 \%)$ & $17260(-5.8 \%)$ & $-3141(0.9 \%)$ & $131(-4.0 \%)$ & 47 \\
\hline s9 & 0.21 & 799 & 0.47 & 19700 & -2967 & 147 & 34 & $0.49(3.0 \%)$ & $18580(-5.7 \%)$ & $-2867(3.4 \%)$ & $141(-4.0 \%)$ & 38 \\
\hline $\mathrm{s} 10$ & 0.22 & 899 & 0.51 & 21000 & -2830 & 157 & 57 & $0.53(3.7 \%)$ & $20580(-2.0 \%)$ & $-2782(1.7 \%)$ & $155(-1.1 \%)$ & 69 \\
\hline $\mathrm{r} 1$ & 1.32 & 267 & 3.79 & 110000 & -4955 & 838 & 69 & $3.97(4.8 \%)$ & $104180(-5.3 \%)$ & $-4844(2.3 \%)$ & $811(-3.2 \%)$ & 27 \\
\hline r2 & 2.60 & 598 & 7.32 & 212760 & -6148 & 1625 & 0 & $7.74(5.7 \%)$ & $202840(-4.7 \%)$ & $-6031(1.9 \%)$ & $1582(-2.6 \%)$ & 71 \\
\hline $\mathrm{r} 3$ & 3.37 & 862 & 9.33 & 275760 & -7358 & 2103 & 102 & $9.89(6.1 \%)$ & $261180(-5.3 \%)$ & $-7297(0.8 \%)$ & $2038(-3.1 \%)$ & 91 \\
\hline $\mathrm{r} 4$ & 6.81 & 1903 & 18.90 & 554260 & -10748 & 4233 & 170 & $19.83(4.9 \%)$ & $522980(-5.6 \%)$ & $-10592(1.4 \%)$ & $4086(-3.5 \%)$ & 175 \\
\hline r5 & 10.20 & 3101 & 28.16 & 823100 & -11984 & 6297 & 256 & $29.48(4.7 \%)$ & $777920(-5.5 \%)$ & $-11804(1.5 \%)$ & $6084(-3.4 \%)$ & 271 \\
\hline & & & & & & & & $(5.0 \%)$ & $(-4.9 \%)$ & $(1.6 \%)$ & $(-3.0 \%)$ & \\
\hline
\end{tabular}

and leads to larger total area of buffers over SBWF. Reduced buffer area in SBWF also leads to $3.0 \%$ reduction of power on average over SBW + Fill. This is in stark contrast to costaware [32] and power-optimal wire-sizing and buffering [24], [29] where the tradeoffs between timing optimality and costs (e.g., buffer/wiring area, power) are much stronger. We also notice that the runtime also slightly increases from SBW + Fill to SBWF due to the evaluation of dishing and erosion model. However, note that the runtime reported in SBW + Fill is for a single run; in practice, designers have to perform multiple runs in order to minimize overdesigning the slew rate constraint $\eta$ as explained earlier, which may therefore cost much more runtime. From all of these results, we see that designs considering CMP impacts improve timing over nominal design, and is not prohibitively expensive in run-time as long as effects of CMP on parasitics is modeled accurately and efficiently.

\section{YIELD-DRIVEN SBW}

\section{A. Leff Variation}

One of the most important process uncertainties that affects circuit performance is the random variation of devices' effective channel lengths $\left(L_{\text {eff }}\right)$ [4], [33] The variation of $L_{\text {eff }}$ manifests itself in changing various device characteristics, e.g., input capacitance $C_{\mathrm{in}}$, effective output resistance $R_{\text {eff }}$, and intrinsic delay $d_{\text {buf }}$. To understand the effect of $L_{\text {eff }}$ variation on the delay, we show two sets of measurements on buffers using SPICE [34]. We model $L_{\text {eff }}$ with a Gaussian distribution $\Delta_{L}$ with its mean value $\overline{L_{\text {eff }}}$ equals its nominal value and the standard deviation $\widehat{L_{\text {eff }}}$ equals $5 \% \cdot \overline{L_{\text {eff }}} \cdot 7$

The first set studies the sensitivity of the effective input capacitance of buffers to $L_{\text {eff }}$ variation. We set the total $L_{\text {eff }}$ of the transistors at the input of an inverter to an unlikely large value and show that the increase in the input capacitance as a consequence is small. We size the PMOS and the NMOS

${ }^{7}$ ITRS [15] allows a budget of $10 \%$ from the nominal value for $3 \times$ standard deviations of random variation (excluding all systematic variation like acrosschip linewidth variations). Other related works in literature [11], [13] assume this budget to be $15 \%-30 \%$. of the buffers to the ratio of $2: 1$ for symmetric rise and fall. Therefore, the total input capacitance is a function of $L_{\text {eff }}^{\text {tot }}=$ $L_{\text {eff }}^{n}+2 \cdot L_{\text {eff }}^{p}$, where $L_{\text {eff }}^{n}$ and $L_{\text {eff }}^{p}$ are the $L_{\text {eff }}$ of the NMOS and PMOS transistors, respectively. Since $L_{\text {eff }}^{n}$ and $L_{\text {eff }}^{p}$ are assumed to be independent Gaussian random variables having the same Gaussian distribution $\Delta_{L}, L_{\text {eff }}^{\text {tot }}$ is also a Gaussian random variable with mean $3 \cdot \overline{L_{\text {eff }}}$ and standard deviation $\sqrt{5} \cdot \widehat{L_{\mathrm{eff}}}$. The $99 \%$ percentile of $L_{\mathrm{eff}}^{\text {tot }}$ is given by

$$
L_{\text {eff }}^{\alpha}=\sqrt{5} \cdot \operatorname{CDF}_{\text {Gaussian }}^{-1}(0.99) \cdot \widehat{L_{\mathrm{eff}}}+3 \cdot \overline{L_{\mathrm{eff}}}
$$

where $\mathrm{CDF}_{\mathrm{Gaussian}}^{-1}(x)$ is the inverse Gaussian $\mathrm{CDF}$, and $\operatorname{Prob}\left(L_{\text {eff }}^{\text {tot }}>L_{\text {eff }}^{\alpha}\right) \leq 1 \%$. We first employ the simplified model from [35] that the transistor gate capacitance $C_{g}$ operated in saturation region is given by

$$
C_{g}=C_{\mathrm{ox}} \cdot W_{d} \cdot\left(\frac{2}{3} \cdot L_{\mathrm{eff}}+2 \cdot L_{\mathrm{int}}\right)
$$

where $C_{\mathrm{ox}}$ is the gate oxide capacitance per unit area, $W_{d}$ is the drawn transistor width, and $L_{\text {int }}$ is the length of lateral diffusion. According to the default values in the BSIM $465-\mathrm{nm}$ device model [30], we set $\overline{L_{\text {eff }}}=33 \cdot 3=99 \mathrm{~nm}$ and $L_{\text {int }}=$ $16 \cdot 3=48 \mathrm{~nm}$. We apply (2) to obtain $L_{\text {eff }}^{\alpha}=(99+8.58) \mathrm{nm}$. Using (3), we find that the capacitance increases by only $3.5 \%$ when $L_{\text {eff }}^{\text {tot }}$ increases from $3 \cdot \overline{L_{\text {eff }}}$ to $L_{\text {eff }}^{\alpha}$. To verify this, we increase the $L_{\text {eff }}^{\text {tot }}$ of the transistors to from $3 \cdot \overline{L_{\text {eff }}}$ to $L_{\text {eff }}^{\alpha}$ in SPICE, from which we find that the measured effective input capacitance only increases by less than $3 \%$ for all sizes of buffers in our experiment. This is equivalent to a negligibly small $4.1 \mathrm{fF}$ increase in the input capacitance for our largest $(120 \times)$ buffer. Therefore, we conclude that the effective input capacitance is rather insensitive to random $L_{\text {eff }}$ variation and we treat it as constant in this paper without much loss of accuracy.

The second set of measurement shows that $L_{\text {eff }}$ variation has a much larger contribution to the variation of the effective output resistance $R_{\text {eff }}$ and the intrinsic delay $d_{\text {buf }}$. To account for the dependence of $R_{\text {eff }}$ and $d_{\text {buf }}$ on the common variation source $L_{\text {eff }}$, we model the variation in $R_{\text {eff }}$ and $d_{\text {buf }}$ using a 
joint distribution, which can be obtained from Monte Carlo simulation with SPICE. We collect the covariance matrix as a statistical metric to observe the variability of $R_{\text {eff }}$ and $d_{\text {buf }}$ under $L_{\text {eff }}$ variation, which is given by

$$
M=\left[\begin{array}{ll}
\zeta_{R, R} & \zeta_{R, d} \\
\zeta_{R, d} & \zeta_{d, d}
\end{array}\right]=\left[\begin{array}{cc}
771 & 26.5 \\
26.5 & 14.0
\end{array}\right]
$$

Equation (4) shows the covariance matrix $M$ of a $20 \times$ buffer, where $\zeta_{x, y}$ is the covariance of $x, y \in\{R, d\}$, and subscripts $R$ and $d$ refer to $R_{\text {eff }}$ and $d_{\text {buf }}$, respectively. The standard deviations of $R_{\text {eff }}\left(\sqrt{\zeta_{R, R}}\right)$ and $d_{\text {buf }}\left(\sqrt{\zeta_{d, d}}\right)$ are about $15 \%$ and $6 \%$ of their mean values, respectively. This shows that $R_{\text {eff }}$ and $d_{\text {buf }}$ can deviate significantly from their respective nominal values due to $L_{\text {eff }}$ variation. Moreover, the large covariance between $R_{\text {eff }}$ and $d_{\text {buf }}$ (i.e., $\zeta_{R, d}$ ) demonstrates that $R_{\text {eff }}$ and $d_{\text {buf }}$ are positively correlated, which means that an occurrence of positive (negative) variation in $R_{\text {eff }}$ from the nominal value is likely to be accompanied by a positive (negative) variation in $d_{\text {buf }}$ [36]. Therefore, we characterize $R_{\text {eff }}$ and $d_{\text {buf }}$ using a joint probability density function (pdf) $f_{R, d}\left(R_{\text {eff }}, d_{\text {buf }}\right)$, which accurately models the occurrence probability of the $\left(R_{\text {eff }}, d_{\text {buf }}\right)$ pair, and can be computed by Monte Carlo simulation. Let us consider the delay of a buffer driving a capacitance $C_{L}$, which is given by

$$
d=C_{L} \cdot R_{\mathrm{eff}}+d_{\mathrm{buf}}
$$

in the deterministic case. We substitute (5), into $f_{R, d}\left(R_{\text {eff }}, d_{\text {buf }}\right)$ and then integrate $f_{R, d}$ over $R_{\text {eff }}$ to obtain the pdf of the loaded buffer delay, which is given by

$$
f_{d}\left(C_{L}, d\right)=\int_{-\infty}^{\infty} f_{R, d}\left(R_{\mathrm{eff}}, d-C_{L} \cdot R_{\mathrm{eff}}\right) d R_{\mathrm{eff}}
$$

\section{B. vSBWF Problem Formulation}

We call the SBWF problem, considering $L_{\text {eff }}$ random variation, vSBWF. Owing to its statistical nature, we treat the RAT at each node as a random variable. The objective of vSBWF becomes maximizing a routing tree's statistical timing yield. The timing yield is defined as

$$
\Upsilon=\operatorname{Prob}\left(\mathrm{RAT}_{\mathrm{s}} \geq \Gamma_{\Upsilon}\right)
$$

where $\Gamma_{\Upsilon}$ is the yield cutoff point at $\Upsilon \cdot 100 \%$. This equation essentially says that the probability of $\mathrm{RAT}_{\mathrm{s}}$ at the source $n_{\mathrm{src}}$ being at least $\Gamma_{\Upsilon}$ is $\Upsilon$.

As an important step toward runtime control in any contemporary buffer insertion algorithms [16], [24], [37], pruning using any nominal value such as mean or worst case values, is deficient when timing is subject to random variations. To illustrate, suppose that we are evaluating the merging node $n_{\mathrm{m}}$ where two identical subtrees join. Two buffering solutions from each subtree are propagated to $n_{\mathrm{m}}$, which have discrete delay distributions of $\operatorname{sol}_{A}=(50 \% \cdot 200 \mathrm{ps}, 50 \% \cdot 300 \mathrm{ps})$ and $\operatorname{sol}_{B}=(80 \% \cdot 242.5,20 \% \cdot 305 \mathrm{ps})$, respectively. Let us assume for now that these two solutions do not differ in other metrics (for example, downstream capacitance). We are interested in finding out how each of the pruning metrics (mean, worst case, statistical) picks their solution among all produced at $n_{\mathrm{m}}$. By enumeration, we obtain the following solutions at $n_{\mathrm{m}}$ :

1) $\operatorname{sol}_{\alpha}=\max \left(\operatorname{sol}_{A}, \operatorname{sol}_{A}\right)=(25 \% \cdot 200 \mathrm{ps}, 75 \% \cdot 300 \mathrm{ps})$ : mean $=250 \mathrm{ps}$, worst case $=300 \mathrm{ps}$;

2) $\operatorname{sol}_{\beta}=\max \left(\operatorname{sol}_{A}, \operatorname{sol}_{B}\right)=(40 \% \cdot 242.5 \mathrm{ps}, 40 \% \cdot 300 \mathrm{ps}$, $20 \% \cdot 305)$ : mean $=255$ ps, worst case $=305$ ps;

3) $\operatorname{sol}_{\gamma}=\max \left(\operatorname{sol}_{B}, \operatorname{sol}_{B}\right)=$ $(64 \% \cdot 242.5 \mathrm{ps}, 36 \% \cdot 305 \mathrm{ps})$ : mean $=255 \mathrm{ps}$, worst case $=305$ ps.

Among these solutions, it is clear that all pruning strategies based on nominal values (mean, worst case) prefer $\operatorname{sol}_{\alpha}$ as it has the smallest delay in both metrics. However, we notice that both solutions $\operatorname{sol}_{\alpha}$ and $\operatorname{sol}_{\beta}$ have a much higher proportion of $\geq 300$ ps instances than $\operatorname{sol}_{\gamma}$. Therefore, the merged solution $\mathrm{sol}_{\gamma}$ is considered a statistically superior solution, in which pruning using statistical timing shall be able to identify. It is also possible that statistical pruning may help improve the mean timing of the optimized statistical distribution. For example, the statistical timing distribution of $\operatorname{sol}_{\gamma}$ has a mean of $265 \mathrm{ps}$, while those of $\operatorname{sol}_{\alpha}$ and $\operatorname{sol}_{\beta}$ have means of 275 and 278 ps, respectively.

There are two challenges in solving the vSBWF problem which are: 1) how to efficiently represent and compute RAT that is not a deterministic value but a random variable and 2) how to define pruning rules that remove statistically inferior solutions while keeping the algorithm tractable. We address these challenges in the following sections.

\section{Representing and Computing RAT}

To solve vSBWF via the same DP framework as shown in Section III-B, we have to replace the deterministic RAT computation with its statistical counterpart. Since a random variable can be completely characterized by its CDF, we choose to base all statistical computation in terms of $\mathrm{RAT}_{\text {sol }}^{i}$ 's CDF in any solution $\operatorname{sol}_{i} .{ }^{8}$ We represent $\mathrm{CDF}$ in the form of piecewiselinear curve (PWL) as in [38]. Representing CDF in the form of PWL has the advantage that operations on a complicated function become a series of operations on ramp functions, which often have closed-form solutions. For example, using PWL reduces statistical addition and maximum operations to convolution of steps and ramps and multiplication of ramps, respectively, both of which have closed-form quadratic solutions. Reference [38] has depicted operations for Elmore delay calculation and has provided closed-form quadratic formulas. After all operations on these ramp and step functions, summing the resulting quadratic curves forms a "piecewise quadratic curve." This curve is then "sampled" at the predefined percentile to produce the final CDF in the PWL.

The application of PWL is not limited to the first-order delay and slew models used in this paper. Our immediate observation is that the PWL model can also apply to at least second-order models. For example, delay and slew rate metrics in [26] and

\footnotetext{
${ }^{8}$ In our implementation, we consider the negative of $\mathrm{RAT}_{\mathrm{sol}}$, i.e., $-\mathrm{RAT}_{\text {sol }}$, for the sake of simpler mathematical manipulation. This converts ball "min" operations at branch merging points into "max" operations, which are equivalent to simple multiplications of CDFs in the statistical domain.
} 
[27] require the computation of the second moment. The second moment qcomputation involves multiplication of two independent random variables and squaring of random variables, both of which can be expressed analytically. By modeling CDFs with PWL curves, we can find the analytical solution for each PWL component and proceed with the same methodology to compute CDFs.

\section{Efficient Pruning in $v S B W F$}

A useful pruning rule must: 1) not discard any partial solution that may lead to the optimal solution sol opt $_{\text {at }}$ at the source $n_{\text {src }}$ and 2) keep the growth of number of solutions polynomial with respect to the tree size. We propose an efficient yield cut-off dominance-pruning heuristic. This heuristic provably keeps the solutions' growth at a linear rate. Although we cannot prove analytically that such heuristic preserves the optimal solution, we experimentally show that the optimality of the solution's timing is comparable to the CDF dominance-pruning rule, which is provably optimal but leads to exponential runtime.

1) CDF Dominance: Fig. 7(a) shows the CDF dominance relationship. Area CDF 1 is completely on the right-hand side of CDF 2. As a result, CDF 2 is said to be dominated and is discarded under this relationship. To see why pruning under this relationship preserves optimality, we show mathematically that $\widetilde{\mathrm{CDF}}_{1}(x)$ and $\widetilde{\mathrm{CDF}}_{2}(x)$ computed from $\mathrm{CDF}_{1}(x)$ and $\mathrm{CDF}_{2}(x)$ in delay and slew rate computations have the same relative superiority as $\mathrm{CDF}_{1}(x)$ and $\mathrm{CDF}_{2}(x)$. Suppose that $\mathrm{CDF}_{1}(x) \geq \mathrm{CDF}_{2}(x) \forall x$. Statistical maximum corresponds to CDF multiplication, which is obtained by

$$
\begin{aligned}
\widetilde{\mathrm{CDF}}_{1}(x) & =\mathrm{CDF}_{1}(x) \cdot \mathrm{CDF}(x) \\
& \geq \mathrm{CDF}_{2}(x) \cdot \mathrm{CDF}(x) \\
& =\widetilde{\mathrm{CDF}}_{2}(x)
\end{aligned}
$$

since $\operatorname{CDF}(x)$ is nonnegative. Statistical addition corresponds to the convolution of CDF and pdf, which is

$$
\widetilde{\operatorname{CDF}}_{i}(x)=\int_{-\infty}^{\infty} \operatorname{CDF}_{i}(\tau) \cdot \operatorname{pdf}(x-\tau) d \tau
$$

where $i=1,2$ and $\operatorname{pdf}(x)=(d / d x) \operatorname{CDF}(x)$. Since $\mathrm{CDF}_{1}(x)-\mathrm{CDF}_{2}(x) \geq 0$ and $\operatorname{pdf}(x) \geq 0 \quad \forall x$, we have

$$
\begin{aligned}
\int_{-\infty}^{\infty}\left(\mathrm{CDF}_{1}(\tau)-\mathrm{CDF}_{2}(\tau)\right) & \cdot \operatorname{pdf}(x-\tau) d \tau \\
= & \widetilde{\mathrm{CDF}_{1}}(x)-\widetilde{\mathrm{CDF}}_{2}(x) \geq 0
\end{aligned}
$$

and therefore we have $\widetilde{\mathrm{CDF}}_{1}(x) \geq \widetilde{\mathrm{CDF}_{2}}(x)$ again. However, this dominance relationship does not establish a total order among all $\mathrm{RAT}_{\text {sol }}$ because one curve does not dominate another if they cross in the shaded area of Fig. 7(a). Therefore, the pruning effect is weak.

2) Yield Cut-Off Dominance: It is clear from Fig. 7(b) that we only use the yield cutoff $\Gamma_{\Upsilon}$ for comparing the CDFs of the RATs. Since $\Gamma_{1}>\Gamma_{2}$, CDF 1 is said to dominate CDF 2.

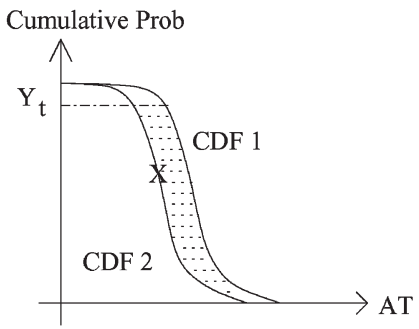

(a)

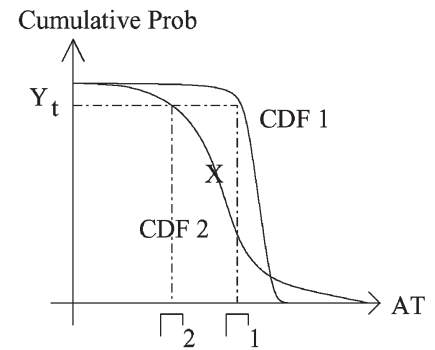

(b)
Fig. 7. CDF of RATs to illustrate the definition of timing yield, yield cutoff point, and pruning rules.

Under this rule, the relative dominance between all pair of curves is well defined, therefore all options are totally ordered. This preserves the property that for each distinct value of load, we only need to retain one solution (which has the largest $\left.\Gamma_{\Upsilon}\right)$. Following from the complexity analysis in Section III-B, the number of distinct capacitance values are tightly upper bounded and hence the number of nondominating solutions is bounded by $O\left(\left|S_{\mathrm{buf}}\right| \cdot c_{\max } \cdot|V|\right)$, where $\left|S_{\mathrm{buf}}\right|, c_{\max }$ and $|V|$ are the number of possible buffer sizes, the maximum capacitance value and the number of tree nodes, respectively. We conceive this pruning rule from the observation that we pick the optimum solution sol ${ }_{\text {opt }}$ at the source $n_{\text {src }}$ by finding the largest $\Gamma_{\Upsilon}$ among all solutions at $n_{\mathrm{src}}$. Therefore, it is reasonable to prune solutions at the same yield point $\Upsilon$ at all nodes without considering the part of CDF larger than $\Upsilon$, which is irrelevant to obtaining the optimal solution.

Notice that even though pruning under yield cut-off dominance only compares one point, it is different from corner case designs since we obtain $\Gamma_{\Upsilon}$ from accurate RAT distributions, which are derived from statistical calculation. In the corner case design, we get the worst case RAT from extreme interconnect and buffer parameters. Using such a worst case RAT leads to severe overdesign.

3) Evaluating the Pruning Rules: Fig. 8 shows the log-plot of the runtime trends when straight wires of different lengths undergo the vSBWF algorithm with the two pruning rules. The number of nodes grows linearly with the length of the wire. The figure shows that the runtime from $\mathrm{CDF}$ dominance pruning grows exponentially with respect to the wire length. In contrast, the curve for yield cut-off dominance-pruning plateaus, which shows that the runtime is polynomial with respect to the line length. The algorithm using CDF dominance pruning is able to finish in a reasonable time only for some small test cases but takes over $24 \mathrm{~h}$ for any of the test benches in Section IV-E.

Table IV shows the statistics of solutions produced using the two pruning rules, respectively. We handcraft these test cases so that vSBWF with CDF dominance pruning can finish in hours. It is quite obvious that the yield cut-off dominance pruning loses almost no optimality when used in place of the theoretically delay-optimal CDF dominance pruning. With this observation and the runtime concern, we shall use the yield cut-off dominance pruning in practice and in our subsequent discussion in the experiment section.

To maximize the timing yield $\Upsilon$, the best solution to pick at the source $n_{\text {src }}$ is the one which has the largest yield cutoff 


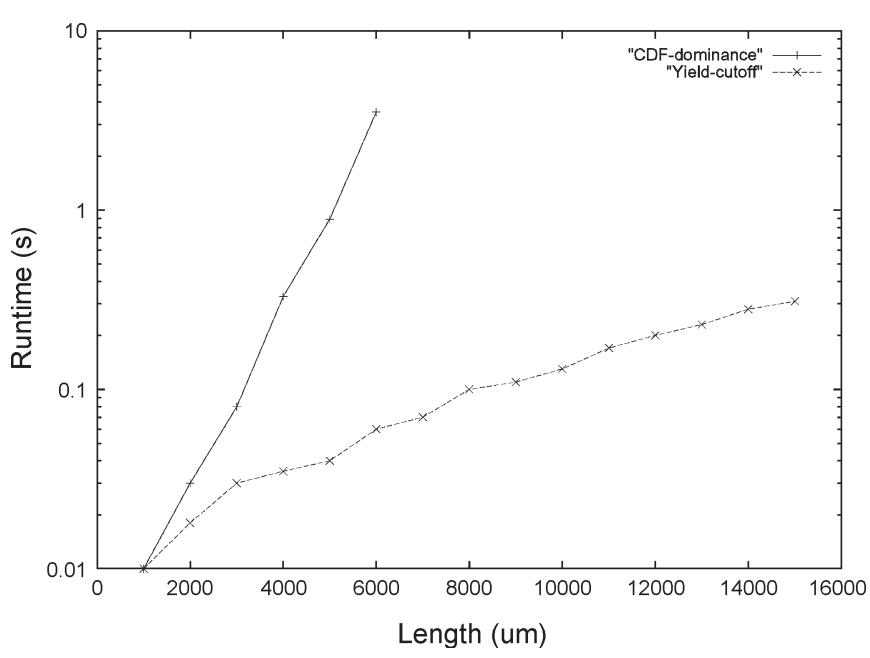

Fig. 8. Runtime in log-scale with different pruning rules.

TABLE IV

COMPARISON BETWEEN PRUNING USING CDF DOMINANCE AND YIELD CUT-OFF DOMINANCE

\begin{tabular}{|c|c|c|c|c|}
\hline & \multicolumn{2}{|c|}{ CDF } & \multicolumn{2}{c|}{ Yield Cut-off } \\
\hline $\begin{array}{c}\text { Test- } \\
\text { bench }\end{array}$ & $\begin{array}{c}\text { Mean } \\
(\mathrm{ps})\end{array}$ & $\begin{array}{c}\text { SD (ps) } \\
(\mathrm{ps})\end{array}$ & $\begin{array}{c}\text { Mean }(\mathrm{ps}) \\
(\Delta \%)\end{array}$ & $\begin{array}{c}\text { SD }(\mathrm{ps}) \\
(\Delta \%)\end{array}$ \\
\hline line & -6569 & 338 & $-6569(0 \%)$ & $338(0 \%)$ \\
\hline 5-sink & -11543 & 505 & $-11545(0 \%)$ & $511(1.2 \%)$ \\
\hline 6-sink & -9189 & 437 & $-9192(0.03 \%)$ & $438(0.002 \%)$ \\
\hline
\end{tabular}

point $\Gamma_{\Upsilon}$. The timing yield $\Upsilon$ can be chosen by designers to fulfill their yield objective.

\section{E. Experiment}

We carry out the experiment on the same test cases in Section III-D. We use SBW + Fill, which reflects the current design methodology, as our baseline case. To show whether any "partial solution" is adequate to address the vSBWF problem, we also compare vSBWF against SBWF from Section III,

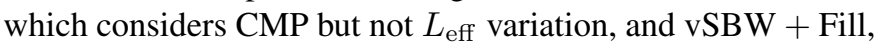
which considers $L_{\text {eff }}$ variation without CMP. The assumptions on $L_{\text {eff }}$ follow from Section IV-A. The vSBWF problem requires a different slew rate constraint due to its random nature, therefore all SBW + Fill, SBWF and vSBW + Fill require different overconstrain rates from the one used in Section III-D. We again rely on the binary search using SBW + Fill, SBWF and $\mathrm{vSBW}+$ Fill to find this new overconstrain rate $\kappa$. We choose the new slew rate constraint to be $\operatorname{Prob}($ slew $\leq \eta) \geq$ $99 \%$ at all inputs of buffers and sinks $t_{i}$, where $\eta=100$ ps. This means that the slew rate at all buffer inputs and sinks $t_{i}$ must have $99 \%$ chance meeting the bound $\eta$. Under this new requirement, we have found that the overconstrain rate $\kappa$ for SBW + Fill, SBWF and vSBW + Fill are 0.75, 0.78, and 0.85 , respectively. In contrast, the vSBWF algorithm considers the random variation during optimization and therefore directly produces optimum solution sol $_{\text {opt }}$ that meet such slew rate constraint. The yield $\Upsilon$ we optimize for is set to 0.9. We use the same computing platform as in Section III-D. To verify the solutions, we perform statistical, CMP-aware timing analysis on the solutions from SBW + Fill, SBWF, vSBW + Fill, and vSBWF through Monte Carlo simulation, which is set to achieve $0.1 \%$ error in mean values with $99 \%$ confidence.

To compare the solutions produced by SBW + Fill, SBWF, vSBW + Fill, and vSBWF in the random $L_{\text {eff }}$ regime, we use the concept of timing yield. To illustrate, Fig. 9 shows the pdfs of the RATs from the optimized solutions on a large net "s10." We use the $90 \%$ yield cutoff point, $\Gamma_{90 \%}$, of the vSBWF's RAT solution, which is $2962 \mathrm{ps}$, as the threshold for timing tests. We regard the proportion of the pdf that has RAT better than $\Gamma_{90 \%}=2962$ ps as yield. In other words, the pdf of vSBWF has a yield rate of $90 \%$ shown in the shaded area under its curve. Similarly, the yield from the pdf of SBW + Fill is $37.7 \%$, while those of SBWF and vSBW + Fill are almost $0 \%$.

Table V shows the comparison between SBW + Fill, SBWF, vSBW + Fill, and vSBWF under both CMP and random $L_{\text {eff }}$ variation. We report the yield of SBW + Fill designs in the fifth column of Table V. SBW + Fill results in a significant $43.1 \%$ yield loss on average compared to the vSBWF designs. We notice that the vSBWF design reduces buffer area in most cases but increases wiring area compared to SBW + Fill. In general, we observe that considering CMP tends to decrease buffer area due to overconstraining slew rate as explained in Section III-D, while considering random $L_{\text {eff }}$ variation tends to increase buffer area for extra design margin. On the other hand, considering either CMP or random variation alone, as in the case of SBWF or vSBW + Fill, does not produce the desired optimal buffering and wire sizing solutions, which are shown by the poor yields in the seventh and the ninth columns. The runtime of vSBWF is roughly $25 \times$ of SBWF. ${ }^{9}$

We also look into the effectiveness of statistical design on the possible increased random variation in the future process technologies. Fig. 10 shows the probability distributions of net " 1 " optimized using SBW + Fill, and vSBWF under the assumption of standard deviation $\widehat{L_{\text {eff }}}=5 \%$ (curves' label suffixed with " 0.05 ") and $10 \%$ (curves' label suffixed with " 0.10 ") of the mean $\overline{L_{\text {eff }}}$, respectively. The curves are much flatter when $\widehat{L_{\text {eff }}}$ increased to $10 \% \cdot \overline{L_{\text {eff }}}$, with the distribution of timing now spans more than 5\% of the mean delay. Moreover, vSBWF is now capable of achieving bigger improvement in timing. The yield improvement of " $r 1$ " using vSBWF over SBW + Fill is reported to be $12 \%$ from Table V under the $5 \% \widehat{L_{\text {eff }}}$ assumption, while that under the $10 \%$ assumption is almost $90 \%$. The nominal delay improvement by vSBWF over SBW + Fill increases from less than $1 \%$ under the 5\% $\widehat{L_{\text {eff }}}$ assumption to more than $5 \%$ under the $10 \%$ assumption. Experiments on other testcases show similar trend. This shows that statistical design methodologies like vSBWF will become more important for timing closure as process variation increases in future technologies.

\section{CONCLUSiON}

In this paper, we have presented extensions to the DP algorithm for simultaneous wire sizing and buffer insertion (SBW) to account for the impacts of CMP-induced and random channel

\footnotetext{
${ }^{9}$ Runtime of s1-s5 are not compared since overhead of initializing PWL calculation dominates the runtime of these small test cases.
} 


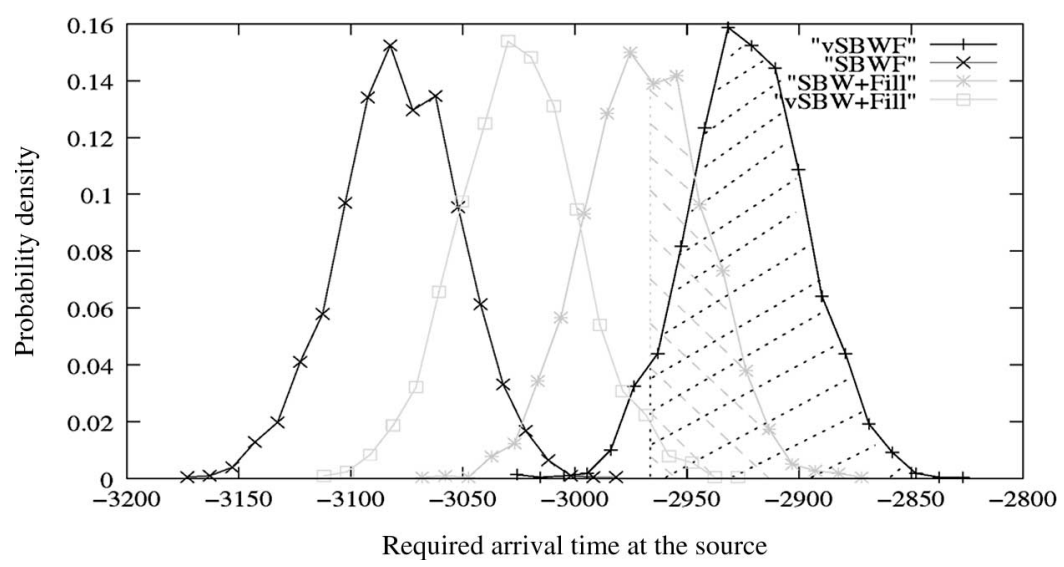

Fig. 9. Probability density distribution of net "s10."

TABLE V

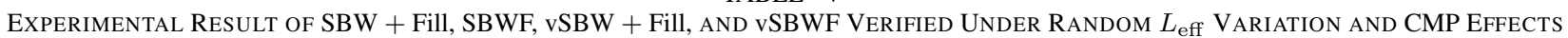

\begin{tabular}{|c|c|c|c|c|c|c|c|c|c|c|c|c|}
\hline & \multicolumn{4}{|c|}{$\begin{array}{c}S B W+F i l l \\
(\kappa=0.75)\end{array}$} & \multicolumn{2}{|c|}{$\begin{array}{c}S B W F \\
(\kappa=0.78)\end{array}$} & \multicolumn{2}{|c|}{$\begin{array}{c}v S B W+\text { Fill } \\
(\kappa=0.85)\end{array}$} & \multicolumn{4}{|c|}{$v S B W F$} \\
\hline $\begin{array}{l}\text { test- } \\
\text { case }\end{array}$ & $\begin{array}{c}\text { wire } \\
\text { area } \\
\left(m^{2} m^{2}\right)\end{array}$ & $\begin{array}{c}\text { buffer } \\
\text { area } \\
\left(10^{3} \times\right)\end{array}$ & $\begin{array}{l}\text { nominal } \\
\text { RAT } \\
(p s)\end{array}$ & $\begin{array}{c}\text { yield } \\
(\%)\end{array}$ & $\begin{array}{c}\text { nominal } \\
\text { RAT }(p s) \\
(\Delta \%)\end{array}$ & $\begin{array}{c}\text { yield } \\
(\%)\end{array}$ & $\begin{array}{c}\text { nominal } \\
\text { RAT }(p s) \\
(\Delta \%)\end{array}$ & $\begin{array}{c}\text { yield } \\
(\%)\end{array}$ & $\begin{array}{c}\text { wire area } \\
\left(m^{2}\right) \\
(\Delta \%)\end{array}$ & $\begin{array}{c}\text { buffer area } \\
\left(10^{3} \times\right) \\
(\Delta \%)\end{array}$ & $\begin{array}{c}\text { nominal } \\
\text { RAT }(p s) \\
(\Delta \%)\end{array}$ & $\begin{array}{c}\text { run- } \\
\text { time } \\
(s)\end{array}$ \\
\hline s1 & 0.10 & 3.3 & -1105 & $12 \%$ & $-1105(0 \%)$ & $6 \%$ & $-1107(-0 \%)$ & $5 \%$ & $0.11(8 \%)$ & $3.2(-1 \%)$ & $-1059(4 \%)$ & 23 \\
\hline s2 & 0.11 & 3.5 & -1176 & $97 \%$ & $-1232(-5 \%)$ & $7 \%$ & $-1177(-0 \%)$ & $93 \%$ & $0.12(7 \%)$ & $3.3(-6 \%)$ & $-1176(0 \%)$ & 28 \\
\hline s3 & 0.14 & 4.9 & -1677 & $90 \%$ & $-1728(-3 \%)$ & $18 \%$ & $-1678(-0 \%)$ & $95 \%$ & $0.15(8 \%)$ & $4.8(-1 \%)$ & $-1676(0 \%)$ & 33 \\
\hline s4 & 0.18 & 6.7 & -1460 & $10 \%$ & $-1533(-5 \%)$ & $0 \%$ & $-1441(1 \%)$ & $49 \%$ & $0.19(7 \%)$ & $6.5(-3 \%)$ & $-1412(3 \%)$ & 77 \\
\hline s5 & 0.26 & 9.7 & -2579 & $93 \%$ & $-2724(-6 \%)$ & $0 \%$ & $-2587(-0 \%)$ & $86 \%$ & $0.29(11 \%)$ & $9.6(-1 \%)$ & $-2579(0 \%)$ & 174 \\
\hline s6 & 0.31 & 12.7 & -2400 & $90 \%$ & $-2516(-5 \%)$ & $0 \%$ & $-2454(-2 \%)$ & $17 \%$ & $0.35(12 \%)$ & $12.7(-0 \%)$ & $-2399(0 \%)$ & 265 \\
\hline s7 & 0.38 & 15.8 & -4024 & $35 \%$ & $-4225(-5 \%)$ & $0 \%$ & $-4083(-1 \%)$ & $1 \%$ & $0.43(12 \%)$ & $16.1(2 \%)$ & $-3967(1 \%)$ & 558 \\
\hline s8 & 0.43 & 19.8 & -3337 & $35 \%$ & $-3464(-4 \%)$ & $0 \%$ & $-3338(-0 \%)$ & $31 \%$ & $0.49(13 \%)$ & $19.3(-3 \%)$ & $-3284(2 \%)$ & 1022 \\
\hline s9 & 0.47 & 21.6 & -3092 & $11 \%$ & $-3174(-3 \%)$ & $0 \%$ & $-3095(-0 \%)$ & $10 \%$ & $0.52(12 \%)$ & $21.3(-1 \%)$ & $-3024(2 \%)$ & 1080 \\
\hline s10 & 0.50 & 22.0 & -2967 & $38 \%$ & $-3078(-4 \%)$ & $0 \%$ & $-3023(-2 \%)$ & $1 \%$ & $0.56(12 \%)$ & $22.8(3 \%)$ & $-2922(2 \%)$ & 1610 \\
\hline $\mathrm{r} 1$ & 3.74 & 116.6 & -5177 & $78 \%$ & $-5604(-8 \%)$ & $0 \%$ & $-5312(-3 \%)$ & $0 \%$ & $4.09(10 \%)$ & $115.9(-1 \%)$ & $-5160(0 \%)$ & 690 \\
\hline r2 & 7.31 & 229.4 & -6511 & $30 \%$ & $-7029(-8 \%)$ & $0 \%$ & $-6715(-3 \%)$ & $0 \%$ & $7.97(9 \%)$ & $226.4(-1 \%)$ & $-6458(1 \%)$ & 1663 \\
\hline r3 & 9.32 & 299.0 & -7716 & $60 \%$ & $-8280(-7 \%)$ & $0 \%$ & $-7989(-4 \%)$ & $0 \%$ & $10.17(9 \%)$ & $295.9(-1 \%)$ & $-7669(1 \%)$ & 2189 \\
\hline $\mathrm{r} 4$ & 18.74 & 596.6 & -11439 & $24 \%$ & $-12369(-8 \%)$ & $0 \%$ & $-11735(-3 \%)$ & $0 \%$ & $20.54(10 \%)$ & $595.8(-0 \%)$ & $-11344(1 \%)$ & 3682 \\
\hline r5 & 28.07 & 895.1 & -12796 & $0 \%$ & $-13830(-8 \%)$ & $0 \%$ & $-13119(-3 \%)$ & $0 \%$ & $30.57(9 \%)$ & $885.2(-1 \%)$ & $-12502(2 \%)$ & 5480 \\
\hline & & & & $47 \%$ & $(-5 \%)$ & $2 \%$ & $(-1 \%)$ & $26 \%$ & $(10 \%)$ & $(-1 \%)$ & $(1 \%)$ & \\
\hline
\end{tabular}

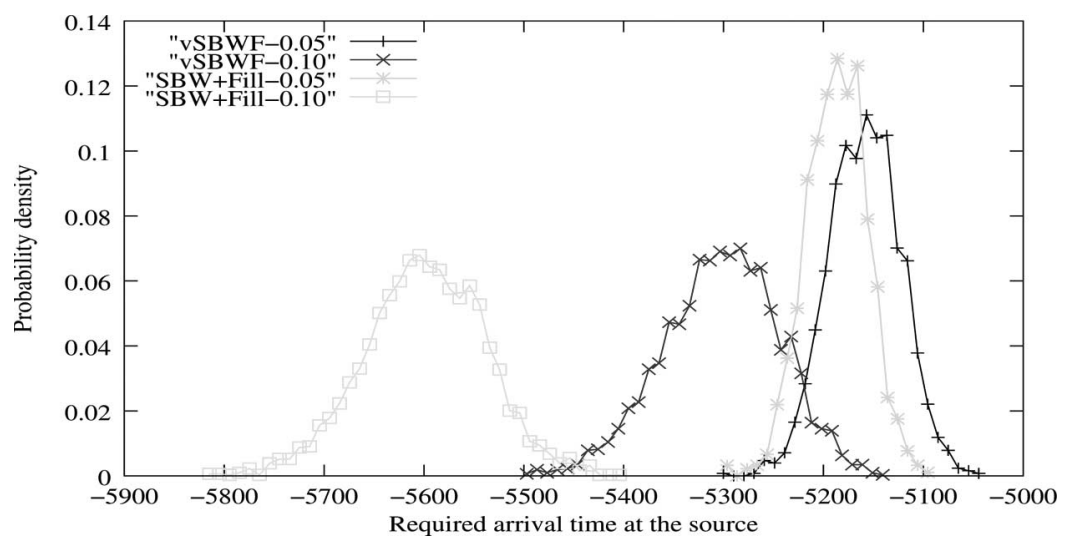

Fig. 10. Probability density distribution of net "r1" assuming $\widehat{L_{\text {eff }}}=5 \%$ and $10 \%$ of $\overline{L_{\text {eff }}}$.

length $\left(L_{\text {eff }}\right)$ process variation on parasitics and timing performance. We have first quantitatively studied the potential impact of CMP-variation on interconnect parasitics, based on which we have developed an accurate, table look-up-based $R C$ model considering systematic CMP variation with precalculated, optimized fill-patterns that minimize coupling capacitance. Based on this, we have studied the simultaneous buffer insertion, wire- sizing, and fill insertion problem (SBWF). Experiment under conservative assumptions on process variation have shown that the proposed SBWF designs consistently achieve 1.6\% delay reduction on average over nominal design $(\mathrm{SBW}+$ Fill $)$. We also approach the SBW problem considering both systematic CMP variation and random $L_{\text {eff }}$ variation (vSBWF) by incorporating efficient statistical timing analysis into the 
SBWF algorithm. We have developed an efficient heuristic for pdf pruning, whose practical optimality is comparable to a provably optimal yet expensive pruning rule. Experimental results show that (vSBWF) increases timing yield by $43.1 \%$ on average, compared to SBW + Fill which considers nominal $L_{\text {eff }}$ value. All these extensions do not change the fundamental DP framework, therefore they are compatible with other extensions that consider power/area-constrained SBW optimization, which have been intensively studied recently.

In this paper, we assume a fixed routing topology with buffer insertion and wire sizing as a postrouting optimization. In the future, we plan to study simultaneous routing topology generation with buffer insertion and wire sizing considering both interconnect and device variations.

\section{REFERENCES}

[1] C. Visweswariah, "Death, taxes and failing chips," in Proc. Des. Autom. Conf., Jun. 2003, pp. 343-347.

[2] Y. Chen, P. Gupta, and A. B. Kahng, "Performance-impact limited area fill synthesis," in Proc. Des. Autom. Conf., Jun. 2003, pp. 22-27.

[3] T. Tugbawa, T. Park, D. Boning, T. Pan, P. Li, S. Hymes, T. Brown, and L. Camilletti, "A mathematical model of pattern dependencies in Cu CMP processes," in Proc. CMP Symp., Electrochem. Soc. Meeting, Oct. 1999, pp. 605-615.

[4] P. Gupta and F. Heng, "Towards a systematic-variation aware timing methodology," in Proc. Des. Autom. Conf., Jun. 2004, pp. 321-326.

[5] B. Stine, D. Boning, J. Chung, L. Camilletti, F. Kruppa, E. Equi, W. Loh, S. Prasad, M. Muthukrishnan, D. Towery, M. Berman, and A. Kapoor, "The physical and electrical effects of metal-fill patterning practices for oxide chemical-mechanical polishing processes," IEEE Trans. Electron Devices, vol. 45, no. 3, pp. 665-679, Mar. 1998.

[6] K.-H. Lee, J.-K. Park, Y.-N. Yoon, D.-H. Jung, J.-P. Shin, Y.-K. Park, and J.-T. Kong, "Analyzing the effects of floating dummy-fills: From feature scale analysis to full-chip RC extraction," in Proc. IEDM Tech. Dig., Dec. 2001, pp. 31.3.1-31.3.4.

[7] W. Grobman, M. Thompson, R. Wang, C. Yuan, R. Tian, and E. Demircan, "Reticle enhancement technology: Implications and challenges for physical design," in Proc. Des. Autom. Conf., 2001, pp. 73-78.

[8] J. Jess, K. Kalafala, S. Naidu, R. Otten, and C. Visweswariah, "Statistical timing for parametric yield prediction of digital integrated circuits," in Proc. Des. Autom. Conf., Jun. 2003, pp. 932-937.

[9] A. Agarwal, D. Blaauw, V. Zolotov, and S. Vrudhula, "Computation and refinement of statistical bounds on circuit delay," in Proc. Des. Autom. Conf., Jun. 2003, pp. 348-353.

[10] C. Visweswariah, K. Ravindran, K. Kalafala, S. Walker, and S. Narayan, "First-order incremental block-based statistical timing analysis," in Proc. Des. Autom. Conf., Jun. 2004, pp. 331-336.

[11] S. Choi, B. Paul, and K. Roy, "Novel sizing algorithm for yield improvement under process variation in nanometer technology," in Proc. Des. Autom. Conf., Jun. 2004, pp. 454-459.

[12] S. Raj, S. Vrudhula, and J. Wang, "A methodology to improve timing yield in the presence of process variation," in Proc. Des. Autom. Conf., Jun. 2004, pp. 448-453.

[13] A. Agarwal, K. Chopra, and D. Blaauw, "Statistical timing based optimization using gate sizing," in Proc. Des. Autom. Test Eur., Mar. 2005, pp. 400-405.

[14] V. Khandelwal, A. Davoodi, A. Nanavati, and A. Srivastava, "A probabilistic approach to buffer insertion," in Proc. Int. Conf. Comput.-Aided Des., Nov. 2003, pp. 560-567.

[15] Semiconductor Industry Association, International Technology Roadmap for Semiconductors, 2003.

[16] L. P. P. P. van Ginneken, "Buffer placement in distributed RC-tree networks for minimal Elmore delay," in Proc. IEEE Int. Symp. Circuits Syst., 1990, pp. 865-868.

[17] J. Cong, L. He, C. Koh, and Z. Pan, "Interconnect sizing and spacing with considering of coupling capacitance," IEEE Trans. Comput.-Aided Design Integr. Circuits Syst., vol. 20, no. 9, pp. 1164-1169, Sep. 2001.

[18] J.-K. Park, K.-H. Lee, J.-H. Lee, Y.-K. Park, and J.-T. Kong, "An exhaustive method for characterizing the interconnect capacitance considering the floating dummy-fills by employing an efficient field solving algorithm," in Proc. SISPAD, Sep. 2000, pp. 98-101.
[19] P. Gupta and A. B. Kahng, "Manufacturing-aware physical design," in Proc. Int. Conf. Comput.-Aided Des., Oct. 2003, pp. 681-687.

[20] R. Tian, X. Tang, and D. Wong, "Dummy-feature placement for chemicalmechanical polishing uniformity in a shallow trench isolation process," IEEE Trans. Comput.-Aided Design Integr. Circuits Syst., vol. 21, no. 1, pp. 63-71, Jan. 2002.

[21] Magam Design Automation Inc., Quickcap User Manual. [Online]. Available: http://www.magma-da.com/

[22] T. E. Gbondo-Tugbawa, "Chip-scale modeling of pattern dependencies in copper chemical mechanical polishing process," $\mathrm{Ph} . \mathrm{D}$. dissertation, Massachusetts Inst. Technol., Cambridge, MA, 2002.

[23] A. Kahng, G. Robins, A. Singh, H. Wang, and A. Zelikovsky, "Filling and slotting: Analysis and algorithms," in Proc. Des. Autom. Test Eur., April 1998, pp. 95-102.

[24] J. Lillis, C. K. Cheng, and T. T. Y. Lin, "Optimal wire sizing and buffer insertion for low power and a generalized delay model," in Proc. Int. Conf. Comput.-Aided Des., Nov. 1995, pp. 138-143.

[25] H. Bakoglu, Circuits, Interconnects and Packaging for VLSI. Reading, MA: Addison-Wesley, 1990.

[26] C. Alpert, D. Devgan, and C. Kashyap, "RC delay metrics for performance optimization," IEEE Trans. Comput.-Aided Design Integr. Circuits Syst., vol. 20, no. 5, pp. 571-582, May 2001.

[27] K. Agarwal, D. Sylvester, and D. Blauuw, "A simple metric for $R C$ circuit based on two circuit moments," IEEE Trans. Comput.-Aided Design Integr. Circuits Syst., vol. 23, no. 9, pp. 1346-1354, 2004.

[28] Z. Li, C. Sze, C. Alpert, J. Hu, and W. Shi, "Making fast buffer insertion even faster via approximation techniques," in Proc. Asia South Pacific Des. Autom. Conf., Jan. 2005, pp. 13-18.

[29] K. Tam and L. He, "Power optimal dual-vdd buffered tree considering buffer stations and blockages," in Proc. Des. Autom. Conf., Jun. 2005, pp. 497-502.

[30] Berkeley Predictive Technology Model. University of California, Berkeley. [Online]. Available: http://www-device.eecs.berkeley.edu/ptm

[31] R.-S. Tsay, "An exact zero-skew clock routing algorithm," IEEE Trans. Comput.-Aided Design Integr. Circuits Syst., vol. 12, no. 2, pp. 242-249, Feb. 1993.

[32] W. Shi, Z. Li, and C. Alpert, "Complexity analysis and speedup techniques for optimal buffer insertion with minimum cost," in Proc. Asia South Pacific Des. Autom. Conf., Jan. 2005, pp. 609-614.

[33] Y. Cao, P. Gupta, A. Kahng, D. Sylvester, and J. Yang, "Design sensitivities to variability: Extrapolations and assessments in nanometer VLSI," in Proc. ASIC/SOC Conf., Sep. 2002, pp. 411-415.

[34] N. Weste and D. Harris, CMOS VLSI Design: A Circuits and Systems Perspective. Reading, MA: Addison-Wesley, 2004.

[35] J. Rabaey, A. Chandrakasan, and B. Nikolic, Digital Integrated CircuitsA Design Perspective. Englewood Cliffs, NJ: Prentice-Hall, 2003.

[36] A. Leon-Garcia, Probability and Random Processes for Electrical Engineering. Reading, MA: Addison-Wesley, 1994.

[37] W. Shi and Z. Li, "An o(nlogn) time algorithm for optimal buffer insertion," in Proc. Des. Autom. Conf., Jun. 2003, pp. 580-585.

[38] A. Devgan and C. Kashyap, "Block-based static timing analysis with uncertainty," in Proc. Int. Conf. Comput.-Aided Des., Nov. 2003, pp. 607-614.

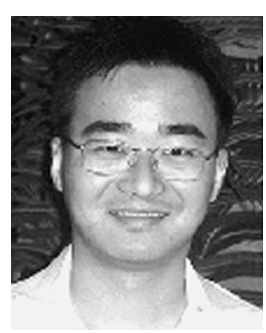

Lei He (S'94-M'99) received the B.S. degree in electrical engineering from Fudan University, Shanghai, China, in 1990, and the Ph.D. degree in computer science from University of California, Los Angeles (UCLA), in 1999.

$\mathrm{He}$ is currently an Assistant Professor with the Electrical Engineering Department, UCLA. From 1999 to 2001 , he was a faculty member with University of Wisconsin, Madison. He held industrial positions with Cadence, Hewlett-Packard, Intel, and Synopsys. His research interests include computeraided design (CAD) of very-large-scale-integration (VLSI) circuits and systems, interconnect modeling and design, programmable logic and interconnect, and power-efficient circuits and systems.

Dr. He received the Dimitris N. Chorafas Foundation Prize for Engineering and Technology in 1997, the Distinguished Ph.D. Award from UCLA Henry Samueli School of Engineering and Applied Science, in 2000, the National Science Foundation (NSF) CAREER award, in 2000, the UCLA Chancellor's Faculty Development Award, in 2003, and the IBM Faculty Award, in 2003. 


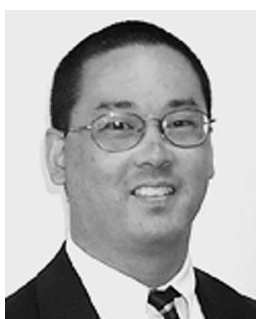

Andrew B. Kahng (M'03) received the A.B. degree in applied mathematics from Harvard University, Cambridge, MA, and the M.S. and Ph.D. degrees in computer science, both from University of California, San Diego, in 1986 and 1989, respectively.

He is a Professor of Computer Science and Engineering and Electrical and Computer Engineering with University of California, San Diego. He has published over 200 papers in the VLSI CAD literature. From 1989 to 2000, he was a member of the University of California at Los Angeles (UCLA) computer science faculty. His research is mainly in the physical design and performance analysis of VLSI as well as the VLSI design manufacturing interface. His other research interests include combinatorial and graph algorithms, and large-scale heuristic global optimization. In 1997, he defined the physical design roadmap for the International Technology Roadmap for Semiconductors (ITRS), and since 2001, he has chaired the U.S. and international working groups for design technology for the ITRS. He has been active in the MARCO Gigascale Silicon Research Center since its inception.

Dr. Kahng is a recipient of three Best Paper awards and an NSF Young Investigator award. He was also the founding General Chair of the Association for Computing Machinery (ACM)/IEEE International Symposium on Physical Design and cofounded the ACM Workshop on System-Level Interconnect Planning.

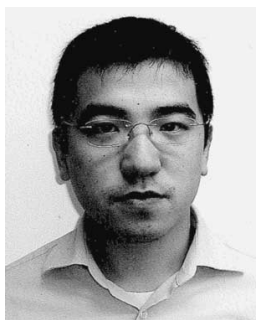

King Ho Tam received the B.E. degree in computer engineering from Hong Kong University of Science and Technology, Clear Water Bay, Hong Kong, in 2002, and the M.Sc. degree in electrical engineering from University of California, Los Angeles (UCLA), in 2005. He is currently working toward the Ph.D. degree with the Design Automation Lab, UCLA.

He was a summer intern in design technology at Intel, CA, in 2003, and with the T. J. Watson Research Center at IBM, NY, in 2005. Since late 2005, he has been with Cadence Design Systems, CA, as a Technical Staff Member. His research interests include low power, process variation-aware physical design problems, and signal integrity analysis.

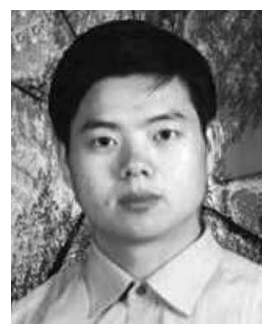

Jinjun Xiong (S'04-M'07) received the B.E. degrees (with honors) in precision instrument and industrial engineering and the M.E. degree in precision instruments, in 1998 and 2000, respectively, from Tsinghua University, China, the M.S. degree in electrical and computer engineering from University of Wisconsin, Madison, in 2001, and the Ph.D. degree in electrical engineering from University of California, Los Angeles (UCLA), in 2006

$\mathrm{He}$ is currently a Research Staff Member with the IBM Thomas J. Watson Research Center, Yorktown Heights, NY. His research interests include statistical timing analysis and optimization, design for manufacturability, design automation for VLSI circuits and systems, large-scale optimization, and combinatorial mathematics.

Dr. Xiong received the Distinguished Graduate Fellowship from the University of Wisconsin, Madison, in 2001, and from UCLA, in 2002. He is the recipient of the Best Student Paper Award at the International Conference on Application-Specific Integrated Circuit 2003, and the Best Paper Award at the ACM International Symposium on Physical Design in 2006. He is also the recipient of the 2005-2006 Outstanding Ph.D. Award in Electrical Engineering from UCLA. 